\title{
Bound state solutions for a class of nonlinear Schrödinger equations
}

\section{Denis Bonheure and Jean Van Schaftingen}

\begin{abstract}
We deal with the existence of positive bound state solutions for a class of stationary nonlinear Schrödinger equations of the form

$$
-\varepsilon^{2} \Delta u+V(x) u=K(x) u^{p}, \quad x \in \mathbb{R}^{N},
$$

where $V, K$ are positive continuous functions and $p>1$ is subcritical, in a framework which may exclude the existence of ground states. Namely, the potential $V$ is allowed to vanish at infinity and the competing function $K$ does not have to be bounded. In the semi-classical limit, i.e. for $\varepsilon \sim 0$, we prove the existence of bound state solutions localized around local minimum points of the auxiliary function $\mathcal{A}=V^{\theta} K^{-\frac{2}{p-1}}$, where $\theta=(p+1) /(p-1)-N / 2$. A special attention is devoted to the qualitative properties of these solutions as $\varepsilon$ goes to zero.
\end{abstract}

\section{Introduction}

The nonlinear Schrödinger equation appears in many fields of physics as nonlinear optics or plasma physics. It typically reads

$$
i \hbar \frac{\partial \psi}{\partial t}+\frac{\hbar^{2}}{2 m} \Delta \psi-W(x) \psi+|\psi|^{p-1} \psi=0, \quad(t, x) \in \mathbb{R} \times \mathbb{R}^{N},
$$

where $\hbar$ denotes the Plank constant and $i$ is the imaginary unit. This equation models the non-relativistic evolution of a quantum particle. It is expected that classical mechanics can be recovered by letting $\hbar \rightarrow 0$ in (1.1) and the limiting behaviour as $\hbar \rightarrow 0$ is then called semi-classical. The

2000 Mathematics Subject Classification: Primary: 35J60. Second.: 35B25, 35B40, 35J10. Keywords: Nonlinear Schrödinger equation, semi-classical states, concentration, vanishing potentials, unbounded competition functions. 
study of the dynamics of (1.1) leads naturally to standing wave solutions, i.e. solutions of the form

$$
\psi(t, x)=e^{-i E t / \hbar} u(x),
$$

where $E$ is the energy of the wave. For small $\hbar$, these solutions are referred to as semi-classical states. The function $\psi$ is a standing wave solution of (1.1) if and only if $u$ solves the semilinear elliptic equation

$$
-\varepsilon^{2} \Delta u+V(x) u=|u|^{p-1} u, \quad x \in \mathbb{R}^{N},
$$

where $\varepsilon^{2}=\hbar^{2} / 2 m$ and $V(x)=(W(x)-E)$.

The study of (1.2) goes back at least to Floer and Weinstein [18] who investigated the special case where $N=1$ and $p=3$. Assuming that $V$ is a globally bounded potential having a nondegenerate critical point, say $x=0$, and $\inf _{\mathbb{R}^{N}} V>0$, they constructed a positive solution $u_{\varepsilon}$ of (1.2) for small $\varepsilon>0$ via a Lyapunov-Schmidt reduction. Moreover, they proved that the solution concentrates around the critical point of $V$, i.e. most of the mass of $u_{\varepsilon}$ is contained in a neighbourhood of 0 that shrinks to a single point as $\varepsilon \rightarrow 0$. This result was then extended to higher dimensions by Oh [26, 28] who also considered the possibility of simultaneous concentration around multiple critical points leading to so-called multi-bump standing waves. Oh [27] also investigated the stability of these solutions.

Since then, equation (1.2) has attracted the interest of many mathematicians and the existence of positive solutions under various assumptions has been proved using different methods. As the problem has generated an impressive amount of publications, it is impossible to give a comprehensive list of references here. Basically, two main routes have been pursued. The Lyapunov-Schmidt reduction scheme proposed by Floer and Weinstein has been further extended and combined with variational arguments by Ambrosetti et al. [1, 7, 4, 5], see also for example [25, 30] for multibump solutions. On the other hand, Rabinowitz [33] initiated a purely variational approach, then mainly relayed by del Pino and Felmer $[14,15,16,17]$. We also refer to P. L. Lions [23], Y. Li [24], Bahri and P. L. Lions [9] as well as to their bibliographies for other works involving variational methods to treat the existence of standing waves for nonlinear Schrödinger equations.

The Lyapunov-Schmidt reduction method introduced by Floer and Weinstein uses in an essential way the nondegeneracy of the critical point of $V$ so that one can address the natural question whether alternative arguments may be used to extend their result to a degenerate setting, that is whether solutions concentrating around possibly degenerate critical points of the potential can be obtained. Using a local variational approach, del Pino 
and Felmer [15, 17] constructed positive solutions concentrating around any topologically nontrivial critical point of the potential $V$ whereas an affirmative answer to the above question has also been given using finite dimensional reduction arguments by Ambrosetti, Badiale and Cingolani [1] for isolated critical points of $V$ with polynomial degeneracy and by Y.Y. Li [25] in the case where $V$ has stable critical points. Basically, the approach of Y.Y. Li requires that small $C^{1}$ perturbations of the potential still have a critical point while that of del Pino and Felmer works fine with any critical point having a minimax characterisation, the easiest situation being that of a local minimum. Assume for instance that $\Lambda \subset \mathbb{R}^{N}$ is a bounded open set such that

$$
V_{0}:=\inf _{x \in \Lambda} V(x)<\inf _{x \in \partial \Lambda} V(x) .
$$

Then, if $\inf _{\mathbb{R}^{N}} V>0$, there exists a positive solution concentrating in $\Lambda$. More precisely, we have the following result which is by now classical.

Theorem (del Pino-Felmer [14]). Assume that $V$ is a locally Hölder continuous potential bounded away from zero and $\Lambda$ is a bounded open set satisfying (1.3). Then, there exist $\varepsilon_{0}>0$ and a family of positive solutions $\left\{u_{\varepsilon} \in H^{1}\left(\mathbb{R}^{N}\right) \mid 0<\varepsilon<\varepsilon_{0}\right\}$ of (1.2) with the particularity that each $u_{\varepsilon}$ possesses a single maximum point $x_{\varepsilon}$ such that $V\left(x_{\varepsilon}\right) \rightarrow V_{0}$ as $\varepsilon \rightarrow 0$. Moreover, there exist $C, \lambda>0$ such that for every $x \in \mathbb{R}^{N}$,

$$
u_{\varepsilon}(x) \leq C \mathrm{e}^{-\lambda\left|x-x_{\varepsilon}\right| / \varepsilon}
$$

and the limiting profile is given by

$$
u_{\varepsilon}(x)=v\left(\frac{x-x_{\varepsilon}}{\varepsilon}\right)+w_{\varepsilon}(x),
$$

where $v$ is the unique positive radial solution of

$$
-\Delta v+V_{0} v=|v|^{p-1} v
$$

and $w_{\varepsilon} \rightarrow 0$ in $C_{\mathrm{loc}}^{2}\left(\mathbb{R}^{N}\right)$ and in $L^{\infty}\left(\mathbb{R}^{N}\right)$ as $\varepsilon \rightarrow 0$.

Formally, equation (1.2) is the Euler-Lagrange equation associated to the action functional

$$
I_{\varepsilon}(u):=J_{\varepsilon}(u)-\frac{1}{p+1} \int_{\mathbb{R}^{N}}|u|^{p+1},
$$

where

$$
J_{\varepsilon}(u):=\frac{1}{2} \int_{\mathbb{R}^{N}}\left(\varepsilon^{2}|\nabla u|^{2}+V(x)|u|^{2}\right) .
$$


Assuming that $1<p<(N+2) /(N-2)$ if $N \geq 3$ and $V(x) \rightarrow+\infty$ as $|x| \rightarrow \infty$, it is easily seen that for any $\varepsilon>0, J_{\varepsilon}$ achieves a minimum constrained to the manifold

$$
\mathcal{M}:=\left\{\left.u \in H^{1}\left(\mathbb{R}^{N}\right)\left|\int_{\mathbb{R}^{N}} V(x)\right| u\right|^{2}<\infty \text { and } \int_{\mathbb{R}^{N}}|u|^{p+1}=1\right\} .
$$

When $\inf _{\mathbb{R}^{N}} V>0$, this allows to obtain a positive ground-state, i.e. a least energy mountain pass solution, of (1.2). In [33], still assuming that $\inf _{\mathbb{R}^{N}} V>0$, Rabinowitz proved the existence of a positive ground state for any $\varepsilon>0$ under the condition

$$
0<a \leq V(x) \leq \liminf _{|x| \rightarrow \infty} V(x), \quad \text { for all } x \in \mathbb{R}^{N} \text { and some } a>0,
$$

with strict inequality on a set of positive measure, while he observed that for small $\varepsilon$, existence holds under the weaker assumption

$$
\inf _{x \in \mathbb{R}^{N}} V(x)<\liminf _{|x| \rightarrow \infty} V(x) .
$$

In the meantime, Wang [37] proved under this last hypothesis that any positive ground state does concentrate at a global minimum point of $V$ as $\varepsilon \rightarrow 0$. These last results concern the case where $V$ achieves a global minimum, i.e. the case where we can choose any sufficiently large bounded set $\Lambda$ in the del Pino-Felmer Theorem. When $V$ only achieves local minima, one requires, from a variational point of view, local arguments to catch local mountain passes. This is the spirit of the approach of del Pino and Felmer [14] which relies on the study of a penalized functional. For instance, the modification in the functional which basically corresponds to a penalization outside $\Lambda$, permits to recover enough compactness to secure the existence of a mountain pass critical point. This critical point is then shown to be a critical point of the original functional when $\varepsilon$ is small.

For various reasons, the penalization scheme developed by del Pino and Felmer crucially depends on the assumption

$$
\inf _{\mathbb{R}^{N}} V>0 .
$$

The case where $\inf _{\mathbb{R}^{N}} V=0$ (this happens if the frequency $E$ of the wave is equal to $\inf _{\mathbb{R}^{N}} W$ which is referred to as the critical frequency [11]) has been treated recently by Byeon and Wang in [11] concerning the existence of ground states and in [12] concerning localized solutions. The results contrast strikingly with the non-critical frequency case since the amplitude of the solutions goes to 0 as $\varepsilon \rightarrow 0$ and the limiting profiles depend on the shape of the potential around the minimum points of $V$. It is worth pointing out that even if the results of Byeon and Wang allow $V$ to vanish in $\mathbb{R}^{N}$, they do require $V$ to be bounded away from zero outside a compact set of $\mathbb{R}^{N}$. 
The starting point of our work is a recent result of Ambrosetti, Felli and Malchiodi [2] where the authors consider the model equation

$$
-\varepsilon^{2} \Delta u+V(x) u=K(x)|u|^{p-1} u, \quad x \in \mathbb{R}^{N},
$$

where $K>0$ is an additional competing function, assumed to be positive. Such an equation has been previously treated by Wang and Zeng [38] assuming that $\inf _{\mathbb{R}^{N}} V>0$ and $K$ is bounded away from zero and bounded. The novelty in [2] is the case where the potential $V$ vanishes at infinity. This situation differs drastically from that considered by Byeon and Wang [11] since the fact that $V$ achieves its infimum at infinity can clearly produce a lack of compactness. Indeed, one easily observes for example that $I_{\varepsilon}$, defined by (1.4), cannot have a least energy mountain pass critical point in this case. In order to recover the existence of a ground state, Ambrosetti, Felli and Malchiodi consider (1.6) with $K(x) \rightarrow 0$ as $|x| \rightarrow \infty$ with a rate related to that of $V$. More precisely, they assume that there exist $A, a, \alpha>0$ and $B, \beta>0$ such that

$$
\frac{a}{1+|x|^{\alpha}} \leq V(x) \leq A \text { and } 0<K(x) \leq \frac{B}{1+|x|^{\beta}} .
$$

Assuming further that $N \geq 3,0<\alpha<2, \beta>0$ and defining

$$
\sigma:=\left\{\begin{array}{cl}
\frac{N+2}{N-2}-\frac{4 \beta}{\alpha(N-2)} & \text { if } \beta<\alpha, \\
1 & \text { if } \alpha \leq \beta
\end{array}\right.
$$

they prove the existence of a positive ground state in $H^{1}\left(\mathbb{R}^{N}\right)$ provided

$$
\sigma<p<\frac{N+2}{N-2}
$$

This condition is sharp in the sense that for this range of $p$, the ground state realizes the supremum

$$
\sup _{\mathcal{H}_{\varepsilon} \backslash\{0\}} \frac{\int_{\mathbb{R}^{N}} K(x)|u|^{p+1}}{\int_{\mathbb{R}^{N}}\left(\varepsilon^{2}|\nabla u|^{2}+V(x)|u|^{2}\right)^{\frac{p+1}{2}}},
$$

where $\mathcal{H}_{\varepsilon}$ is the weighted Sobolev space defined by

$$
\mathcal{H}_{\varepsilon}:=\left\{u \in H_{\mathrm{loc}}^{1}\left(\mathbb{R}^{N}\right) \mid \int_{\mathbb{R}^{N}}\left(\varepsilon^{2}|\nabla u|^{2}+V(x)|u|^{2}\right)<\infty\right\},
$$

while the supremum is $+\infty$ if $p<\sigma$ or $p>(N+2) /(N-2)$. Notice that in case $V$ and $K$ are radial, the range of admissible $p$ 's given by (1.8) can be enlarged when looking for radial ground states as shown recently by $\mathrm{Su}$ et al. [35]. 
In fact, under assumption (1.8), the Sobolev space $\mathcal{H}_{\varepsilon}$ is compactly imbedded in $L^{p+1}\left(\mathbb{R}^{N}, K(x) d x\right)$ so that a positive ground state in $\mathcal{H}_{\varepsilon}$ can be obtained via a classical minimax procedure. At this point, it is worth mentioning that (1.8) implies that the function

$$
\mathcal{A}(x):=[V(x)]^{\frac{p+1}{p-1}-\frac{N}{2}}[K(x)]^{-\frac{2}{p-1}}
$$

tends to $+\infty$ as $|x| \rightarrow \infty$ whereas one can check this last condition actually implies the compact imbedding of $\mathcal{H}_{\varepsilon}$ into $L^{p+1}\left(\mathbb{R}^{N}, K(x) d x\right)$ for any $\varepsilon>0$. The existence condition obtained in [2] can therefore be replaced by just imposing the coercivity of $\mathcal{A}$ without any special decay condition on $V$ and $K$. However, one of the main issues of [2] is in fact to show that this ground state is indeed in $H^{1}\left(\mathbb{R}^{N}\right)$. To this respect, the assumption $\alpha<2$ in (1.7) seems essential.

When $V$ is bounded from below by a positive constant and $K$ is bounded, the auxiliary function $\mathcal{A}$ was shown by Wang and Zeng [38] to play in some sense the role of the potential in Rabinowitz's result, i.e. their extension of Rabinowitz's sufficient condition for the existence of a positive ground state solution of (1.6) with $\varepsilon$ small enough reads

$$
\inf _{x \in \mathbb{R}^{N}} \mathcal{A}(x)<\frac{\liminf |x| \rightarrow \infty}{\lim \sup _{|x| \rightarrow \infty} K(x)^{\frac{p+1}{p-1}-\frac{N}{2}}} .
$$

Under the same conditions, Wang and Zeng also proved that $\mathcal{A}$ contains the information about concentration, namely concentration occurs around critical points of $\mathcal{A}$. Therefore $\mathcal{A}$ is referred to as the concentration function associated to (1.6). In particular, if $V$ is bounded from below by a positive constant and $K$ is bounded, any positive ground state solution of (1.6) concentrates at a global minimum point of $\mathcal{A}$. In fact, one could also rewrite del Pino-Felmer Theorem within the framework of equation (1.6), using the function $\mathcal{A}$ instead of $V$ to locate the peak of the solution. Therefore, the result of [2] can be seen as a partial extension of that theorem in the case of equation (1.6) with both $V$ and $K$ decaying to 0 at infinity and $\mathcal{A}$ having a global minimum.

In this paper, we consider (1.6) in situations where

$$
\liminf _{|x| \rightarrow \infty} \mathcal{A}(x)=0
$$

and

$$
\mathcal{A}(x)>0 \text { for all } x \in \mathbb{R}^{N} \text {. }
$$

This means we consider either a critical frequency case $\inf _{\mathbb{R}^{N}} V=0$, assuming further $V(x)>0$ for all $x \in \mathbb{R}^{N}$, or an unbounded competing function $K$. 
Since in general ground states do not exist in such cases, we search for bound state solutions localized around local minima of $\mathcal{A}$. Our approach relies on a suitable adaptation of the above discussed penalization method of del Pino and Felmer. Our results also concern positive solutions for equations like (1.6) in bounded or exterior domains with Dirichlet boundary condition. In the latter, we assume that the domain has a bounded boundary, $V>0$ in the interior of the domain while we allow $V$ to vanish or $K$ to explode on the boundary.

From now on, $\Omega \subset \mathbb{R}^{N}$ denotes a regular domain with bounded boundary. Of course, this includes the case $\Omega=\mathbb{R}^{N}$. Let $V, K \in C\left(\Omega, \mathbb{R}^{+}\right)$satisfy, if $\partial \Omega \neq \emptyset$

$$
\left(\mathcal{G}_{\partial \Omega}\right) \quad \limsup _{x \rightarrow \partial \Omega} d(x, \partial \Omega)^{p-1} \frac{K(x)}{V(x)}<\infty
$$

and one of the three following growth conditions at infinity if $\Omega$ is unbounded: $\left(\mathcal{G}_{\infty}^{1}\right)$ there exist $\alpha \in[0,2[$ and $\lambda>0$ such that

$$
\liminf _{|x| \rightarrow \infty} V(x)|x|^{\alpha}>0 \text { and } \limsup _{|x| \rightarrow \infty} \psi_{\alpha, \lambda}(x) \frac{K(x)}{V(x)}<\infty,
$$

where $\psi_{\alpha, \lambda}:=\exp \left(-\lambda|x|^{1-\alpha / 2}\right)$;

$\left(\mathcal{G}_{\infty}^{2}\right)$ there exists $\lambda>0$ such that

$$
\liminf _{|x| \rightarrow \infty} V(x)|x|^{2}>0 \text { and } \limsup _{|x| \rightarrow \infty} \psi_{2, \lambda}(x) \frac{K(x)}{V(x)}<\infty,
$$

where $\psi_{2, \lambda}(x):=|x|^{-\lambda}$;

$\left(\mathcal{G}_{\infty}^{3}\right) N>2$ and

$$
\limsup _{|x| \rightarrow \infty}|x|^{-\nu} \frac{K(x)}{V(x)}<\infty,
$$

where $\nu=(p-1)(N-2)$.

Theorem 1. Suppose $\Omega \subset \mathbb{R}^{N}$ is a regular domain with bounded boundary and $p \in] 1,(N+2) /(N-2)[$ if $N \geq 3$ or $p \in] 1, \infty[$ otherwise. Let $V, K \in$ $C\left(\Omega, \mathbb{R}^{+}\right)$satisfy $\left(\mathcal{G}_{\partial \Omega}\right)$ if $\partial \Omega \neq \emptyset$ and one set $\left(\mathcal{G}_{\infty}^{i}\right)$ of growth conditions if $\Omega$ is unbounded. Let $\Lambda \subset \mathbb{R}^{N}$ be open and bounded and assume

$$
\inf _{x \in \Lambda} \mathcal{A}(x)<\inf _{x \in \partial \Lambda} \mathcal{A}(x),
$$

where $\mathcal{A}$ is defined by (1.9). 
Then there exists $\varepsilon_{0}>0$ such that for every $0<\varepsilon<\varepsilon_{0}$, the Dirichlet problem

$$
\begin{aligned}
-\varepsilon^{2} \Delta u+V(x) u & =K(x)|u|^{p-1} u, \quad x \in \Omega, \\
u(x) & =0, \quad x \in \partial \Omega
\end{aligned}
$$

has at least one positive solution $u_{\varepsilon}$.

We emphasize that the solution $u_{\varepsilon}$ also satisfies

$$
\int_{\Omega}\left(\varepsilon^{2}\left|\nabla u_{\varepsilon}\right|^{2}+V(x)\left|u_{\varepsilon}\right|^{2}\right)=O\left(\varepsilon^{N}\right),
$$

but since $V$ does not have a positive lower bound, $\left\|u_{\varepsilon}\right\|_{L^{2}(\Omega)}^{2}$ does not need to satisfy the same estimate, and might even not be finite. However, the solution displays the following features.

Theorem 2. Under the assumptions of Theorem 1 , let $u_{\varepsilon}$ be the solution of (1.11) obtained in that theorem and $x_{\varepsilon} \in \Omega$ be such that

$$
u_{\varepsilon}\left(x_{\varepsilon}\right)=\sup _{x \in \Omega} u_{\varepsilon}(x) .
$$

Then, we have

$$
\mathcal{A}\left(x_{\varepsilon}\right) \rightarrow \inf _{x \in \Lambda} \mathcal{A}(x), \quad \text { as } \varepsilon \rightarrow 0,
$$

for every $r>0$ and $\varepsilon$ sufficiently small, $u_{\varepsilon}$ has no local maximum outside the ball $B\left(x_{\varepsilon}, \varepsilon r\right)$ and satisfies

$$
u_{\varepsilon}(x) \leq C \frac{d(x, \partial \Omega)}{1+d(x, \partial \Omega)} \frac{\varepsilon^{N-2}}{\left(\varepsilon^{2}+\left|x-x_{\varepsilon}\right|^{2}\right)^{\frac{N-2}{2}}} .
$$

If moreover,

$$
\liminf _{d(x, \partial \Omega) \rightarrow 0} V(x)>0 \quad \text { and } \quad \liminf _{|x| \rightarrow \infty} V(x)|x|^{2}>0,
$$

then, for every $\lambda>0$, there is $C>0$ and $\varepsilon_{0}>0$ such that if $0<\varepsilon<\varepsilon_{0}$,

$$
u_{\varepsilon}(x) \leq C \frac{d(x, \partial \Omega)}{1+d(x, \partial \Omega)} \frac{\varepsilon^{\lambda}}{\left(\varepsilon^{2}+\left|x-x_{\varepsilon}\right|^{2}\right)^{\frac{\lambda}{2}}},
$$

while if

$$
\left.\liminf _{d(x, \partial \Omega) \rightarrow 0} V(x)>0 \text { and } \liminf _{|x| \rightarrow \infty} V(x)|x|^{\alpha}>0 \text { for some } \alpha \in\right] 0,2[,
$$

then for every $\lambda>0$, there is $C>0$ and $\varepsilon_{0}>0$ such that if $0<\varepsilon<\varepsilon_{0}$,

$$
u_{\varepsilon}(x) \leq C \frac{d(x, \partial \Omega)}{1+d(x, \partial \Omega)} \exp \left(-\lambda\left|\frac{x-x_{\varepsilon}}{\varepsilon}\right|^{1-\alpha / 2}\right) .
$$

When $\Omega=\mathbb{R}^{N}$ the preceding holds provided $d(x, \partial \Omega) /(1+d(x, \partial \Omega))$ is replaced by 1 . 
This theorem implies in particular that $u_{\varepsilon} \in L^{2}(\Omega)$ and $\left\|u_{\varepsilon}\right\|_{2}^{2}=O\left(\varepsilon^{N}\right)$

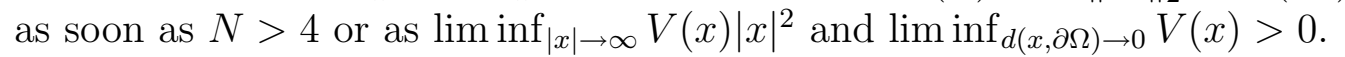

Theorem 2 can be seen as a weak version of the concentration behaviour described in del Pino-Felmer Theorem. If $V$ and $K$ are locally Hölder continuous, then the solution can be shown to achieve a unique (hence global) maximum point. On the other hand, as discussed below, one cannot hope to obtain better decay estimates since they do depend in an essential way on the behaviour of $V$ at infinity.

While completing our research, we heard about some recent preprints dealing also with nonlinear Schrödinger equations with potentials vanishing at infinity. The most related one, by Ambrosetti, Malchiodi and Ruiz [6], concerns the model equation (1.6) in $\mathbb{R}^{N}$ under assumptions similar to ours. Namely, the authors assume therein that $V, K$ are smooth and positive, $V^{\prime}$, $K, K^{\prime}$ are bounded and $V$ decays to zero at infinity in such a way that

$$
\liminf _{|x| \rightarrow \infty} V(x)|x|^{2}>0 .
$$

Under these assumptions, the authors are able to construct, for sufficiently small $\varepsilon$, bound state solutions concentrating at any isolated stable stationary point of the concentration function $\mathcal{A}$. However, their assumptions on the potentials $V$ and $K$ are more restrictive and they require $K$ to be bounded.

Also, the method of [6] relies crucially on the homogeneity of the nonlinear term $K(x) u^{p}$ while we are able to deal with more general nonlinearities. In [6], the authors point out the paper of Souplet and Zhang [34] where radial decaying potentials are considered under stronger growth restrictions at infinity. Moreover, neither semiclassical states nor spikes are investigated.

In two other recent preprints by Ambrosetti and Ruiz [8] and Byeon and Wang [13], the possibility of concentration on spheres in the framework of decaying potentials has also been considered. It could be interesting to see how far our method can be adapted to the search of such solutions. Finally, we point out the note [3], where recent developments on nonlinear Schrödinger equations are discussed. The results of this paper were partially announced in [10].

Our paper is organized as follows. We deal in fact with (1.6) where the nonlinear term $u^{p}$ may be replaced by a more general superlinear term $f(u)$. It is not usually possible to give an explicit expression of the concentration function, i.e. the energy associated the ground state solutions of the limiting equation, see Section 3, which is given by $\mathcal{A}$ in the homogeneous case. Also, the growth conditions $\left(\mathcal{G}_{\infty}^{i}\right)$ have to be adapted according to the behaviour of $f(u) / u$ close to zero. We refer to Section 2 where we complete our general assumptions. We investigate the qualitative properties of the concentration function and those of the ground states of the limiting problem in Section 3. 
In Section 4, we discuss the penalization scheme of del Pino and Felmer and we provide an adequate modification of their approach which works fine within our framework. From the way our penalized functional is defined, it might seem at first sight that the method of del Pino and Felmer extends to our framework with only minor changes. Whereas this is true for some arguments, the possibility of $V$ to vanish at infinity brings a lot of troubles and requires careful estimates. Firstly, our definition of the penalized functional requires some preliminary technical adjustments in order to catch a mountain-pass solution $u_{\varepsilon}$ of the penalized equation.

Indeed, our assumptions on $V$ do not imply the $L^{2}$-boundedness of PalaisSmale sequences as in [14], but Hardy's inequality can still prevent losses of mass at infinity in those sequences. Since Hardy's inequality does not hold in two dimensions, a special care is needed in that case and the compactness of Palais-Smale sequences is derived from a Hardy type inequality with potential which takes a growth restriction on $V$ at infinity into account. This is one of the reasons for which we require $N>2$ when dealing with the weaker growth condition in $\left(\mathcal{G}_{\infty}^{3}\right)$.

To recover a solution of the original equation, we argue as follows. As in [14], for small $\varepsilon$, the solution $u_{\varepsilon}$ is first shown to be small on the boundary of $\Lambda$. This is done in Section 5. Then, using comparison arguments, the estimate on the boundary is extended outside $\Lambda$. To this respect, the situation here is much more delicate than in [14].

Indeed, we do require precise estimates on the decay of the solution at infinity. Such estimates are worked out in Section 6 where the proof of Theorem 1 is completed. Theorem 2 concerning concentration is proved in Section 7. Here again the approach and the results differ considerably due to both weak regularity assumptions on the potentials and their behaviour at infinity.

In [14], when $V$ is Hölder continuous, it is established that $u_{\varepsilon}$ has a unique local (and hence global) maximum. As we only assume that $V$ and $K$ are continuous, the weakness of the regularity of the solution ruled out the arguments used therein.

However, as stated in Theorem 2, the global maximum $x_{\varepsilon}$ is shown to be essentially unique in the sense that if $y_{\varepsilon}$ is a local maxima of $u_{\varepsilon}$, then $d\left(x_{\varepsilon}, y_{\varepsilon}\right)=o(\varepsilon)$ as $\varepsilon \rightarrow 0$. Observe also that when $V$ is not bounded away from zero, we do not recover an exponential decay as in del Pino-Felmer Theorem.

In some sense, the solution inherits its decay properties from the behaviour of $V$. Decay estimates are delicate and depend on comparison arguments uniform in $\varepsilon$. They are obtained by using families of barrier functions, i.e. families of comparison functions having adequate properties. Finally, Section 8 is devoted to some concluding remarks and possible extensions of our results. 


\section{Assumptions}

For further references, we now introduce the main assumptions that will be used throughout the paper. As already mentioned, we deal in fact with the more general Dirichlet problem

$$
\left\{\begin{aligned}
-\varepsilon^{2} \Delta u+V(x) u & =K(x) f(u) & & \text { in } \Omega, \\
u & =0 & & \text { on } \partial \Omega,
\end{aligned}\right.
$$

under the assumptions described hereafter.

\subsection{The domain}

We assume the domain $\Omega \subset \mathbb{R}^{N}$ is a connected open set with bounded $C^{1, \alpha}$ boundary. In the sequel, such domains are referred to as bounded or exterior domains and unless explicitely stated, a regular domain is understood as a domain with $C^{1, \alpha}$ boundary. This regularity assumption is sufficient for our purpose but can be weakened for some pointwise statements.

The boundedness of the boundary is basically used in order to have a uniform control on its geometry. Our arguments would thus also work for some cylindrical domains.

\subsection{The nonlinearity}

We deal with a nonlinearity which is a continuous function $f: \mathbb{R}^{+} \rightarrow \mathbb{R}^{+}$ such that

$\left(f_{1}\right) f(s)=o(s)$ as $s \rightarrow 0^{+}$

$\left(f_{2}\right)$ there exists $1<p<\frac{N+2}{N-2}$ if $N \geq 3$ or $1<p<+\infty$ if $N=1,2$, such that

$$
\lim _{s \rightarrow \infty} \frac{f(s)}{s^{p}}=0 ;
$$

$\left(f_{3}\right)$ there exists $2<\theta \leq p+1$ such that

$$
0<\theta F(s) \leq f(s) s \text { for } s>0,
$$

where $F(s):=\int_{0}^{s} f(\sigma) d \sigma ;$

$\left(f_{4}\right)$ the function $s \mapsto f(s) / s$ is nondecreasing.

We extend $f$ by zero for $s<0$, so that every nonzero solution of $(2.1)$ is by the maximum principle a positive solution of (2.1). 
Combining $\left(f_{1}\right)$ and $\left(f_{2}\right)$, we infer that for every $\delta>0$, there exists $C_{\delta}>0$ such that

$$
f(u) \leq \delta|u|+C_{\delta}|u|^{p} .
$$

Assumption $\left(f_{3}\right)$ implies that there is $C>0$ such that $F(s) \geq C\left(|s|^{\theta}-1\right)$, i.e. $F$ grows superquadratically fast. This condition could be weakened by assuming only that $\left(f_{3}\right)$ holds for large $s$ and for any $a, b>0$, there exists a positive least-energy solution (see below for a precise definition) of the equation

$$
-\Delta u+a u=b f(u) .
$$

The hypothesis $\left(f_{4}\right)$ may also be weakened provided one can still obtain a suitable minimax characterization of the infimum level on the Nehari manifold, see Lemma 3 below. For instance, Wang and Zeng [38] treated a superlinear term of the form $u^{p}-u^{q}$, assuming $q<p$.

Our regularity assumption on the nonlinear term $f$ is weaker than the usual one, see e.g. [14]. Indeed, assuming $f$ of class $C^{1}$ provides quite useful information about the ground states of (2.2): by the symmetry result of Gidas, Ni and Nirenberg [20], any such solution is radial and radially decreasing. This analysis remains valid when $f$ is Lipschitz in a neighbourhood of 0 , see [19]. In the general case, the fact that $u \in H^{1}\left(\mathbb{R}^{N}\right)$ is a ground state and $f$ is nondecreasing implies that $u$ is radial and radialy decreasing, see [36].

\subsection{The potentials}

We next consider two potentials $V, K \in C\left(\Omega, \mathbb{R}^{+}\right)$such that $V(x)>0$. Moreover, we assume $K$ is not identically 0 . If $\Omega$ is unbounded, we impose one of the three following sets of growth conditions at infinity:

$\left(\mathcal{G}_{f, \infty}^{1}\right)$ there exist $\alpha \in[0,2[$ and $\lambda>0$ such that

$$
\liminf _{|x| \rightarrow \infty} V(x)|x|^{\alpha}>0 \text { and } \limsup _{|x| \rightarrow \infty} \frac{f\left(\exp \left(-\lambda|x|^{1-\alpha / 2}\right)\right)}{\exp \left(-\lambda|x|^{1-\alpha / 2}\right)} \frac{K(x)}{V(x)}<1 ;
$$

$\left(\mathcal{G}_{f, \infty}^{2}\right)$ there exists $\lambda>0$ such that

$$
\liminf _{|x| \rightarrow \infty} V(x)|x|^{2}>0 \text { and } \limsup _{|x| \rightarrow \infty} \frac{f\left(|x|^{-\lambda}\right)}{|x|^{-\lambda}} \frac{K(x)}{V(x)}<1 ;
$$

$\left(\mathcal{G}_{f, \infty}^{3}\right) \quad N>2$ and there exists $\lambda>0$ such that

$$
\limsup _{|x| \rightarrow \infty} \frac{f\left(\lambda|x|^{2-N}\right)}{\lambda|x|^{2-N}} \frac{K(x)}{V(x)}<1 .
$$


If the boundary $\Omega$ is not empty, then we also assume that $V$ and $K$ satisfy a growth condition on the boundary:

$\left(\mathcal{G}_{f, \partial \Omega}\right)$ there exist $\mu>0$ such that

$$
\limsup _{d(x, \partial \Omega) \rightarrow 0} \frac{f(\mu d(x, \partial \Omega))}{\mu d(x, \partial \Omega)} \frac{K(x)}{V(x)}<1 .
$$

Observe that all growth conditions depend on the behaviour of $K$ and $V$ at infinity and around $\partial \Omega$, and on the behaviour of $f(s) / s$ around $s=0$. Notice also that only $V$ is assumed to be strictly positive inside $\Lambda$.

\section{Limit problem}

In this section, we introduce the concentration function which is the counterpart of the function $\mathcal{A}$ in the homogeneous case and we study some of its properties. We also provide an independent decay estimate on the ground states of the autonomous limiting problem. We first define the functional $\mathcal{F}_{a, b}: H^{1}\left(\mathbb{R}^{N}\right) \rightarrow \mathbb{R}$ by

$$
\mathcal{F}_{a, b}(u):=\frac{1}{2}\left(\int_{\mathbb{R}^{N}}|\nabla u|^{2}+a \int_{\mathbb{R}^{N}}|u|^{2}\right)-b \int_{\mathbb{R}^{N}} F(u) .
$$

A necessary condition for $u \in H^{1}\left(\mathbb{R}^{N}\right)$ to be a nontrivial critical point of $\mathcal{F}_{a, b}$ is to belong to the Nehari manifold

$$
\mathcal{N}_{a, b}:=\left\{u \in H^{1}\left(\mathbb{R}^{N}\right) \mid u \neq 0,\left(\mathcal{F}_{a, b}^{\prime}(u), u\right)=0\right\} .
$$

We then say that $u \in H^{1}\left(\mathbb{R}^{N}\right)$ is a least-energy solution of

$$
-\Delta u+a u=b f(u)
$$

or equivalently that $u$ is a least-energy critical point of $\mathcal{F}_{a, b}$ if

$$
\mathcal{F}_{a, b}(u)=\inf _{u \in \mathcal{N}_{a, b}} \mathcal{F}_{a, b}(u)
$$

We may now define the ground-energy function

$$
\mathcal{E}: \mathbb{R}_{0}^{+} \times \mathbb{R}_{0}^{+} \rightarrow \mathbb{R}^{+}:(a, b) \mapsto \mathcal{E}(a, b):=\inf _{u \in \mathcal{N}_{a, b}} \mathcal{F}_{a, b}(u)
$$

as the action of any least-energy critical point of $\mathcal{F}_{a, b}$ and consequently we introduce the concentration function $\mathcal{C}: \mathbb{R}^{N} \rightarrow \mathbb{R}^{+} \cup\{+\infty\}$ by

$$
\mathcal{C}(\xi):=\left\{\begin{array}{cl}
\mathcal{E}(V(\xi), K(\xi)) & \text { if } K(\xi)>0 \\
+\infty & \text { otherwise }
\end{array}\right.
$$

In other words, if $K(\xi)>0, \mathcal{C}(\xi)$ is the ground-energy associated to leastenergy solutions of

$$
-\Delta u+V(\xi) u=K(\xi) f(u)
$$




\subsection{Energy of the ground state}

The following lemma provides some basic properties of the ground-energy function. We sketch the proof for completeness.

Lemma 3. Assume $f: \mathbb{R}^{+} \rightarrow \mathbb{R}^{+}$is a continuous function that fulfils assumptions $\left(f_{1}\right)-\left(f_{4}\right)$. Then, for every $(a, b) \in \mathbb{R}_{0}^{+} \times \mathbb{R}_{0}^{+}, \mathcal{E}(a, b)$ is a critical value of $\mathcal{F}_{a, b}$ and we have

$$
\mathcal{E}(a, b)=\inf _{\substack{u \in H^{1}\left(\mathbb{R}^{N}\right) \\ u \neq 0}} \max _{t \geq 0} \mathcal{F}_{a, b}(t u)
$$

If $u \in \mathcal{N}_{a, b}$ and $\mathcal{E}(a, b)=\mathcal{E}(u)$, then $u \in C^{1}$ and up to a translation, $u$ is a radial function such that $\nabla u(x) \cdot x<0$ for every $x \in \mathbb{R}^{N} \backslash\{0\}$.

Moreover, the following properties hold:

(i) the ground-energy function is continuous in $\mathbb{R}_{0}^{+} \times \mathbb{R}_{0}^{+}$;

(ii) for every $b^{*} \in \mathbb{R}_{0}^{+}, a \rightarrow \mathcal{E}\left(a, b^{*}\right)$ is strictly increasing;

(iii) for every $a^{*} \in \mathbb{R}_{0}^{+}, b \rightarrow \mathcal{E}\left(a^{*}, b\right)$ is strictly decreasing;

(iv) for every $\lambda>0, \mathcal{E}(\lambda a, \lambda b)=\lambda^{1-N / 2} \mathcal{E}(a, b)$;

(v) if $f(u)=u^{p}$ with $1<p<(N+2) /(N-2)$ if $N \geq 3$ or $1<p<+\infty$ if $N=1,2$, then

$$
\mathcal{E}(a, b)=\mathcal{E}(1,1) a^{\frac{p+1}{p-1}-\frac{N}{2}} b^{-\frac{2}{p-1}}
$$

Proof. The proof of the minimax characterization of $\mathcal{E}(a, b)$ is by now classical, see e.g. [39]. The key ingredient in the proof is the monotonicity assumption $\left(f_{4}\right)$. Once this characterization is established, it is also quite easy to show that $\mathcal{E}(a, b)$ is a critical value.

These two facts have been essentially proved by Rabinowitz [33]. By classical regularity estimates, $u$ is continuously differentiable. If $f$ is Lipschitz, by $[20,19]$, up to a translation $u$ is radial and $\nabla u \cdot x<0$. When $f$ is monotone, [36] implies that $u$ is radial and $\nabla u \cdot x<0$. For $x \in \mathbb{R}^{N} \backslash\{0\}$, let $H$ denote the halspace whose boundary is perpendicular to $x$ and let $v$ denote the reflection of $u$ with respecto to $\partial H$. By assumption, $u-v \geq 0$ in $H$ with equality on $\partial H$. By the monotonicity of $f,-\Delta(u-v)+a(u-v) \geq 0$. Since $v$ is radial, $u \neq v$, so that by the strong maximum principle, $\nabla u \cdot x<0$.

Let us now focus on the properties $(i)-(v)$.

Property (i). Let $(a, b) \in \mathbb{R}^{+} \times \mathbb{R}^{+}$be given. We first claim that $\mathcal{E}$ is lower semi-continuous. Assume $\left(a_{n}, b_{n}\right) \rightarrow(a, b)$ as $n \rightarrow \infty$ and denote by $u_{n} \in \mathcal{N}_{a_{n}, b_{n}}$ a least-energy solution of

$$
-\Delta u+a_{n} u=b_{n} f(u) .
$$


We infer from assumption $\left(f_{3}\right)$ that there exist $\delta>0$ and $C>0$ such that

$$
\delta\left\|u_{n}\right\|_{H^{1}\left(\mathbb{R}^{N}\right)}^{2} \leq \theta \mathcal{F}_{a_{n}, b_{n}}\left(u_{n}\right)-\left(\mathcal{F}_{a_{n}, b_{n}}^{\prime}\left(u_{n}\right),\left(u_{n}\right)\right) \leq C .
$$

Hence, there exists $u \in H^{1}\left(\mathbb{R}^{N}\right)$ such that, passing to a subsequence if necessary, $u_{n} \rightarrow u$ weakly in $H^{1}\left(\mathbb{R}^{N}\right)$. It then follows from Strauss' compact imbeddings, see e.g. [39], that $u_{n}$ converges strongly in $L^{p}\left(\mathbb{R}^{N}\right)$ for any $p \in] 2,(N+2) /(N-2)[$.

On the other hand, since $u_{n}$ solves (3.2), assumption $\left(f_{2}\right)$ and Sobolev's inequality imply the existence of $c>0$ such that

$$
c \leq\left\|u_{n}\right\|_{H^{1}\left(\mathbb{R}^{N}\right)} .
$$

We now conclude that $u$ is a nontrivial solution of

$$
-\Delta u+a u=b f(u),
$$

so that $u \in \mathcal{N}_{a, b}$ and we deduce from standard arguments that

$$
\mathcal{E}(a, b) \leq \mathcal{F}_{a, b}(u) \leq \liminf _{n \rightarrow \infty} \mathcal{F}_{a_{n}, b_{n}}\left(u_{n}\right)
$$

Since $\mathcal{F}_{a_{n}, b_{n}}\left(u_{n}\right)=\mathcal{E}\left(a_{n}, b_{n}\right)$, the claim follows.

We next prove $\mathcal{E}$ is upper semi-continuous. Let $u \in \mathcal{N}_{a, b}$ be a least-energy solution of (3.2). Consider the function $g(t)=\mathcal{F}_{a, b}(t u)$ on $[0, \infty[$. It follows from $\left(f_{1}\right)$ and $\left(f_{3}\right)$ that $g(0)=0, g(t)>0$ for small $t>0$ and $g(t)<0$ for large $t$. Combining these facts with $\left(f_{4}\right)$, we deduce that $t=1$ is the unique maximum point of $g$. In particular, we have

$$
\mathcal{F}_{a, b}(u)=\max _{t \geq 0} \mathcal{F}_{a, b}(t u) .
$$

Assume now $\left(a_{n}, b_{n}\right) \rightarrow(a, b)$ as $n \rightarrow \infty$. By the minimax characterization of $\mathcal{E}\left(a_{n}, b_{n}\right)$ and from what precedes, we infer that for each $n \geq 0$, there exists a unique $t_{n}$ such that

$$
\mathcal{E}\left(a_{n}, b_{n}\right) \leq \max _{t \geq 0} \mathcal{F}_{a_{n}, b_{n}}(t u)=\mathcal{F}_{a_{n}, b_{n}}\left(t_{n} u\right) .
$$

We now claim that $t_{n} \rightarrow 1$. Notice that the uniqueness of the maximum point of $g$ and the definition of the sequence $\left(t_{n}\right)_{n}$ implies the claim follows as soon as we prove that $\left(t_{n}\right)_{n}$ is bounded and bounded away from 0 . Observe first that $\left(t_{n}\right)_{n}$ is bounded. Indeed, we have $a_{n} \leq \bar{a}, b_{n} \geq \underline{b}$ and if $t_{n} \rightarrow+\infty$ as $n \rightarrow \infty$, we deduce by $\left(f_{3}\right)$ that for $n$ large enough,

$$
\mathcal{F}_{a_{n}, b_{n}}\left(t_{n} u\right) \leq \mathcal{F}_{\bar{a}, \underline{b}}\left(t_{n} u\right)<0,
$$

which is absurd. 
We next prove that $t_{n}$ remains bounded away from zero. Indeed, this follows from the superquadraticity of $F$ which implies that for every $\varepsilon>0$, there exists $C_{\varepsilon}>0$ such that

$$
\mathcal{F}_{a_{n}, b_{n}}\left(t_{n} u\right) \geq t_{n}^{2}\left(\|\nabla u\|_{L^{2}\left(\mathbb{R}^{N}\right)}^{2}+\left(a_{n}-\varepsilon b_{n}\right)\|u\|_{L^{2}\left(\mathbb{R}^{N}\right)}^{2}\right)-C_{\varepsilon} t_{n}^{p}\|u\|_{L^{p}\left(\mathbb{R}^{N}\right)}^{p} .
$$

Hence, since $\varepsilon$ is arbitrary and there exist $\underline{a}, \bar{b}>0$ such that $a_{n} \geq \underline{a}, b_{n} \leq \bar{b}$, this last inequality shows that $t_{n}$ remains bounded away from zero. We are now in a position to conclude the proof. Letting $n$ go to infinity in (3.5), we deduce that

$$
\limsup _{n \rightarrow \infty} \mathcal{E}\left(a_{n}, b_{n}\right) \leq \mathcal{E}(a, b)
$$

Indeed, writing

$$
\mathcal{F}_{a_{n}, b_{n}}\left(t_{n} u\right)=\left(\mathcal{F}_{a_{n}, b_{n}}\left(t_{n} u\right)-\mathcal{F}_{a, b}\left(t_{n} u\right)\right)+\mathcal{F}_{a, b}\left(t_{n} u\right)
$$

we observe that the first term in the right hand side tends to 0 because $\left(t_{n} u\right)_{n}$ is bounded in $H^{1}\left(\mathbb{R}^{N}\right)$ while taking into account that $t_{n} u \rightarrow u$ in $H^{1}\left(\mathbb{R}^{N}\right)$, we deduce that

$$
\mathcal{F}_{a, b}\left(t_{n} u\right) \rightarrow \mathcal{F}_{a, b}(u)
$$

Properties (ii) and (iii). Let $b^{*} \in \mathbb{R}_{0}^{+}$be fixed and assume $u \in H^{1}\left(\mathbb{R}^{N}\right)$ is a least energy critical point of $\mathcal{F}_{a, b^{*}}$. Assume $\underline{a}<a$. Consider again the function $g(t)=\mathcal{F}_{\underline{a}, b^{*}}(t u)$ on $[0, \infty[$. As in (i), we infer there exists $\bar{t}>0$ such that

$$
\mathcal{F}_{\underline{a}, b^{*}}(\bar{t} u)=\max _{t \geq 0} \mathcal{F}_{\underline{a}, b^{*}}(t u) \geq \mathcal{E}\left(\underline{a}, b^{*}\right) .
$$

We now deduce that

$$
\begin{aligned}
\mathcal{E}\left(\underline{a}, b^{*}\right) & \leq \mathcal{F}_{a, b^{*}}(\bar{t} u)+(\underline{a}-a) \int_{\mathbb{R}^{N}} \vec{t}^{2}|u|^{2} \\
& \leq \max _{t \geq 0} \mathcal{F}_{a, b^{*}}(t u)+(\underline{a}-a) \int_{\mathbb{R}^{N}} \vec{t}^{2}|u|^{2} \\
& <\mathcal{E}\left(a, b^{*}\right),
\end{aligned}
$$

so that (ii) follows. The property (iii) follows arguing in a similar way.

Property (iv). Let $\lambda>0$ and for every $u \in H^{1}\left(\mathbb{R}^{N}\right)$, write $u_{\lambda}(x)=u\left(\lambda^{1 / 2} x\right)$. Then, one has

$$
\mathcal{F}_{\lambda a, \lambda b}\left(u_{\lambda}\right)=\lambda^{1-N / 2} \mathcal{F}_{a, b}(u)
$$

Since $u \mapsto u_{\lambda}$ is a bijection of $H^{1}\left(\mathbb{R}^{N}\right)$, this implies $\mathcal{E}(\lambda a, \lambda b)=\lambda^{1-N / 2} \mathcal{E}(a, b)$. 
Property (v). Let $\mu>0$. For every $u \in H^{1}\left(\mathbb{R}^{N}\right)$, one has

$$
\mathcal{F}_{a, \mu b}\left(\mu^{-1 /(p-1)} u\right)=\mu^{-2 /(p-1)} \mathcal{F}_{a, b}(u),
$$

so that $\mathcal{E}(a, \mu b)=\mu^{-2 /(p-1)} \mathcal{E}(a, b)$. Hence, the conclusion follows by computing

$$
\mathcal{E}(a, b)=\mathcal{E}\left(a \cdot 1, a \cdot \frac{b}{a} \cdot 1\right)=a^{\frac{p+1}{p-1}-\frac{N}{2}} b^{-\frac{2}{p-1}} \mathcal{E}(1,1) .
$$

It follows from that lemma and the continuity of $V$ and $K$ that $\mathcal{C}$ is continuous from $\Omega \rightarrow \mathbb{R}^{+} \cup\{\infty\}$. When assuming more regularity on $V$ and $K$, the concentration function can be shown to be of class $C^{1}$ on the set where $\mathcal{C}$ is finite, see [38]. If we also assume that the positive radial ground state of (3.4) is unique for every $\xi$ such that $K(\xi)>0$, then $\mathcal{C}(\xi)$ contains all the information about possible concentration points. Indeed, Wang and Zheng proved that in this case, bound state solutions concentrating on a single point must have their peak converging to a critical points of $\mathcal{C}$. We refer to [38] for a precise statement and to [31] for a similar result in a more general framework.

\subsection{Decay of the ground state}

We next derive a pointwise estimate for ground states of (3.2) which provides information on the decay at infinity.

Proposition 4. Let $u \in \mathcal{N}_{a, b}$ be such that

$$
\mathcal{E}(a, b)=\mathcal{F}_{a, b}(u),
$$

then, up to a translation, $u$ is radial and radially decreasing. Moreover, for every $x \in \mathbb{R}^{N}$, we have

$$
|u(x)|^{2} \leq C \frac{2 \theta}{\theta-2} \frac{\mathcal{E}(a, b)}{a^{1 / 2}|x|^{(N-1)}}
$$

where $C$ is a positive constant that depends only on the dimension of the space and $\theta$ is given in assumption $\left(f_{3}\right)$.

Proof. As $u$ achieves the infimum on the Nehari manifold, it is a ground state solution [39]. It is therefore radial and radially decreasing [19, 20, 36]. Then, by the inequality of Strauss, see e.g. [39], we infer that

$$
|u(x)|^{2} \leq C \frac{\|\nabla u\|_{2}\|u\|_{2}}{|x|^{N-1}}
$$


At last, since $u \in \mathcal{N}_{a, b}$, we deduce from $\left(f_{3}\right)$ that

$$
\left(\frac{1}{2}-\frac{1}{\theta}\right) \int_{\mathbb{R}^{N}}|\nabla u|^{2}+a|u|^{2} \leq \mathcal{F}_{a, b}(u)=\mathcal{E}(a, b)
$$

so that the conclusion follows.

As already noticed, it is well-known, at least when $f$ is smooth, that ground states decay exponentially at infinity, see Gidas, Ni and Nirenberg [20]. The main point of Proposition 4 is that the inequality (3.6) does only depend on the ground energy and holds uniformly for any ground state. Using regularity theory, one can improve (3.6) and obtain usual exponential decay estimates.

\section{The penalization scheme}

In this section, motivated by the paper of del Pino and Felmer [14], we consider a modified problem which is the starting point for a local variational analysis. We first focus on a suitable functional framework.

Formally, the elliptic equation in (2.1) is the Euler-Lagrange equation of the functional

$$
\mathcal{I}_{\varepsilon}(u):=\frac{1}{2} \int_{\Omega}\left(\varepsilon^{2}|\nabla u|^{2}+V(x)|u|^{2}\right)-\int_{\mathbb{R}^{N}} K(x) F(u) .
$$

It is quite natural to consider the functional $\mathcal{I}_{\varepsilon}$ in the weighted Sobolev space

$$
\mathcal{H}_{\varepsilon}:=\left\{u \in \mathcal{D}_{0}^{1,2}(\Omega) \mid \int_{\Omega}\left(\varepsilon^{2}|\nabla u|^{2}+V(x)|u|^{2}\right) d x<+\infty\right\}
$$

where we recall that $\mathcal{D}_{0}^{1,2}(\Omega)$ is the closure of $C_{c}^{\infty}(\Omega)$ for the $L^{2}$-norm of the gradient. The space $\mathcal{H}_{\varepsilon}$ is a Hilbert space with scalar product and norm respectively defined by

$$
\begin{aligned}
(u \mid v)_{\varepsilon} & :=\int_{\Omega}\left(\varepsilon^{2} \nabla u \cdot \nabla v+V(x) u v\right) d x, \\
\|u\|_{\varepsilon}^{2} & :=\int_{\Omega}\left(\varepsilon^{2}|\nabla u|^{2}+V(x)|u|^{2}\right) d x .
\end{aligned}
$$

However, the assumptions on $V$ and $K$ do not ensure that $\mathcal{H}_{\varepsilon}$ is neither embedded in $H_{0}^{1}(\Omega)$, when $\Omega$ is not bounded, nor in $L^{p+1}(\Omega, K(x) d x)$. In particular $\mathcal{I}_{\varepsilon}$ does not need to be finite for every $u \in \mathcal{H}_{\varepsilon}$ so that $I_{\varepsilon}(u) \in \mathbb{R} \cup\{-\infty\}$. 
Moreover, even if $V$ and $K$ are bounded and bounded away from zero, in which case the above-mentioned embeddings hold and the functional is well-defined in $H_{0}^{1}(\Omega)$, the Palais-Smale condition usually fails without a global assumption like one of those proposed by Rabinowitz [33] and Wang and Zheng [38].

As discussed in the introduction, for small values of $\varepsilon$, Wang and Zheng proved the existence of a positive solution assuming that

$$
\inf _{x \in \mathbb{R}^{N}} \mathcal{A}(x)<\frac{\liminf \operatorname{inf|\rightarrow \infty }_{|x|} V(x)^{\frac{p+1}{p-1}-\frac{N}{2}}}{\lim \sup _{|x| \rightarrow \infty} K(x)^{\frac{2}{p-1}}} .
$$

In fact this condition implies that the Palais-Smale condition holds at the mountain pass level of $\mathcal{I}_{\varepsilon}$ for small $\varepsilon$. Since our assumptions allow $V$ to vanish and $K$ to explode as $|x| \rightarrow \infty$, we cannot tackle the problem via a global variational approach.

In order to bypass these difficulties, we follow the penalization method introduced by del Pino and Felmer [14]. This approach which can be seen as a local variational approach, is well adapted to catch positive bound-states when assuming that $V$ is bounded away from zero. In fact the method requires this last assumption in an essential way. In the next subsection, we improve the penalization scheme by using a different penalized functional.

\subsection{The Penalized functional}

Assume there exists $\Lambda \subset \Omega$ whose closure is compact in $\Omega$ such that

$$
\inf _{x \in \Lambda} \mathcal{C}(x)<\inf _{x \in \partial \Lambda} \mathcal{C}(x),
$$

where $\mathcal{C}$ is the concentration function defined by (3.3). We also assume that $\Lambda$ is chosen in such a way that

$$
\sup _{x \in \Lambda} \mathcal{C}(x)<\infty .
$$

The penalization consists in modifying the superquadratic term in $\mathcal{I}_{\varepsilon}$ outside $\Lambda$. To do so, we first define $\tilde{f}: \Omega \times \mathbb{R}^{+} \rightarrow \mathbb{R}$ by

$$
\tilde{f}(x, s):=\min (\kappa V(x) s, K(x) f(s)),
$$

where $0<\kappa<1$. Then, denoting the characteristic function of the set $\Lambda$ by $\chi_{\Lambda}$, we define $g: \Omega \times \mathbb{R}^{+} \rightarrow \mathbb{R}$ by

$$
g(x, s):=\chi_{\Lambda}(x) K(x) f(s)+\left(1-\chi_{\Lambda}(x)\right) \tilde{f}(x, s) .
$$


From now on, we also use the notation $G(x, s):=\int_{0}^{s} g(x, \sigma) d \sigma$. One may easily check that according to $\left(f_{1}\right)-\left(f_{4}\right)$ and the assumptions on $V$ and $K, g$ is a Carathéodory function satisfying

$\left(g_{1}\right) g(x, s)=o(s)$ as $s \rightarrow 0^{+}$uniformly in compact subsets of $\Omega$;

$\left(g_{2}\right)$ there exists $1<p<\frac{N+2}{N-2}$ if $N \geq 3$ or $1<p<+\infty$ if $N=1,2$, such that

$$
\lim _{s \rightarrow \infty} \frac{g(x, s)}{s^{p}}=0
$$

$\left(g_{3}\right)$ there exist $2<\theta \leq p+1$ and $\kappa \in(0,1)$ such that

$$
0<\theta G(x, s) \leq g(x, s) s \quad \text { for all } x \in \Lambda \text { and any } s>0
$$

and

$$
0 \leq 2 G(x, s) \leq g(x, s) s \leq \kappa V(x) s^{2} \quad \text { for all } x \notin \Lambda \text { and any } s>0
$$

$\left(g_{4}\right)$ the function

$$
s \mapsto \frac{g(x, s)}{s}
$$

is nondecreasing for all $x \in \Omega$.

We are now in a position to introduce the penalized functional

$$
\mathcal{J}_{\varepsilon}(u):=\frac{1}{2}\left(\int_{\Omega} \varepsilon^{2}|\nabla u|^{2}+V(x)|u|^{2}\right)-\int_{\Omega} G(x, u) .
$$

Using classical arguments, we can check that $\left(g_{2}\right)$ and $\left(g_{3}\right)$ imply that $\mathcal{J}_{\varepsilon}$ is well-defined and that $\mathcal{J}_{\varepsilon} \in C^{1}\left(\mathcal{H}_{\varepsilon}, \mathbb{R}\right)$. Moreover its critical points are weak solutions of the boundary value problem

$$
\left\{\begin{aligned}
-\varepsilon^{2} \Delta u+V(x) u & =g(x, u) & & \text { in } \Omega, \\
u & =0 & & \text { on } \partial \Omega .
\end{aligned}\right.
$$

We next show that $\mathcal{J}_{\varepsilon}$ has a mountain pass geometry in $\mathcal{H}_{\varepsilon}$. We first observe that 0 is a local minimum.

Lemma 5. Let $g: \mathbb{R}^{+} \rightarrow \mathbb{R}$ be a Carthéodory function. If $g$ satisfies assumptions $\left(g_{1}\right)-\left(g_{3}\right)$ and $V: \Omega \rightarrow \mathbb{R}^{+}$is a continuous function. Then, the functional $\mathcal{J}_{\varepsilon}$ achieves a local minimum at 0 in $\mathcal{H}_{\varepsilon}$. 
Proof. The proof easily follows from the estimate

$$
\begin{aligned}
\mathcal{J}_{\varepsilon}(u) & =\frac{1}{2}\left(\int_{\Omega} \varepsilon^{2}|\nabla u|^{2}+V(x)|u|^{2}\right)-\int_{\Lambda} G(x, u)-\int_{\Omega \backslash \Lambda} G(x, u) \\
& \geq \frac{1-\kappa}{2}\|u\|_{\varepsilon}^{2}+\int_{\Lambda}\left(\frac{\kappa}{2} V(x)|u|^{2}-G(x, u)\right) .
\end{aligned}
$$

Now, as $V$ is continuous and positive, by classical arguments, see e.g. [32], $\left(g_{1}\right)$ and $\left(g_{2}\right)$ imply

$$
\int_{\Lambda} G(x, u)=o\left(\|u\|_{\varepsilon}^{2}\right), \quad \text { as } u \rightarrow 0 .
$$

Therefore, the conclusion follows from the above estimate.

On the other hand, the infimum of $\mathcal{J}_{\varepsilon}$ is $-\infty$. Indeed, if $0 \neq u \in \mathcal{H}_{\varepsilon}$ has support in $\Lambda$, then

$$
\mathcal{J}_{\varepsilon}(\lambda u) \rightarrow-\infty, \quad \text { as } \lambda \rightarrow+\infty \text {. }
$$

We then define the minimax level

$$
c_{\varepsilon}:=\inf _{\gamma \in \Gamma_{\varepsilon}} \max _{t \in[0,1]} \mathcal{J}_{\varepsilon}(\gamma(t))
$$

where $\Gamma_{\varepsilon}$ is the set of continuous paths

$$
\Gamma_{\varepsilon}:=\left\{\gamma \in C\left([0,1], \mathcal{H}_{\varepsilon}\right) \mid \gamma(0)=0, \mathcal{J}_{\varepsilon}(\gamma(1))<0\right\} .
$$

Now that the minimax setting is established, our next step is to check that $\mathcal{J}_{\varepsilon}$ satisfies some compactness condition. This is the object of the next subsection.

\subsection{Palais-Smale}

We first recall that $\left(u_{n}\right)_{n} \subset \mathcal{H}_{\varepsilon}$ is a Palais-Smale sequence for $\mathcal{J}_{\varepsilon}$ at level $c_{\varepsilon}$ if

$$
\mathcal{J}_{\varepsilon}\left(u_{n}\right) \rightarrow c_{\varepsilon} \text { and } \mathcal{J}_{\varepsilon}^{\prime}\left(u_{n}\right) \rightarrow 0 \text { as } n \rightarrow \infty
$$

We say that $\mathcal{J}_{\varepsilon}$ satisfies the Palais-Smale condition, (PS) in short, if any sequence $\left(u_{n}\right)_{n} \subset \mathcal{H}_{\varepsilon}$ for which $\mathcal{J}_{\varepsilon}\left(u_{n}\right)$ is bounded and $\mathcal{J}_{\varepsilon}^{\prime}\left(u_{n}\right) \rightarrow 0$ as $n \rightarrow \infty$ possesses a convergent subsequence.

The existence of a Palais-Smale sequence at level $c_{\varepsilon}$ follows from standard deformation arguments, see for example [39]. Hence, if $\mathcal{J}_{\varepsilon}$ satisfies (PS), the minimax level $c_{\varepsilon}$ is a critical value of $\mathcal{J}_{\varepsilon}$. In the next lemma, we set $\varepsilon=1$, $\mathcal{H}=\mathcal{H}_{1}$ and $\mathcal{J}=\mathcal{J}_{1}$ to simplify the notations. 
Lemma 6. Let $g: \mathbb{R}^{+} \rightarrow \mathbb{R}$ be a Carathéodory function satisfying $\left(g_{1}\right)-\left(g_{3}\right)$ and $V: \Omega \rightarrow \mathbb{R}^{+}$be a continuous function. If $N=2$ and $\Omega$ is unbounded, assume furthermore that

$$
\liminf _{|x| \rightarrow \infty} V(x)|x|^{2}>0
$$

Then, the functional $\mathcal{J}: \mathcal{H} \rightarrow \mathbb{R}$ satisfies $(P S)$.

A special care is required when $\Omega$ is an unbounded two-dimensional domain, due to the failure of Hardy's inequality in that case. This problem is circumvented thanks to the following preliminary lemma which provide a Hardy type inequality in dimension 2.

Lemma 7. Let $\Omega \subset \mathbb{R}^{2}$ be a regular exterior domain and $V \in C\left(\Omega ; \mathbb{R}^{+}\right)$. If

$$
\liminf _{|x| \rightarrow \infty} V(x)|x|^{2}>0
$$

then there exists $C>0$ such that for every $u \in \mathcal{D}(\Omega)$,

$$
\int_{\Omega} \frac{|u|^{2}}{d_{\Omega}(x)^{2}} \leq C \int_{\Omega}|\nabla u|^{2}+V(x)|u|^{2}
$$

where $d_{\Omega}(x)=d(x, \partial \Omega)$ if $\partial \Omega \neq \emptyset$ and $d_{\Omega}(x)=1+|x|$ in the case where $\Omega=\mathbb{R}^{2}$.

Remark 8. When $N \neq 2$ or $\Omega$ is bounded, then one has the stronger classical Hardy inequality

$$
\int_{\Omega} \frac{|u|^{2}}{d_{\Omega}(x)^{2}} \leq C \int_{\Omega}|\nabla u|^{2}
$$

Moreover, one can take $d_{\mathbb{R}^{N}}(x)=|x|$, see e.g. [22].

Proof of Lemma 7. Let us choose $R>1$ such that $\mathbb{R}^{N} \backslash B(0, R) \subset \Omega$, and

$$
c:=\inf _{|x|>R} V(x)|x|^{2}>0 .
$$

Then, the set $\Omega^{\prime}=\Omega \cap B(0,3 R)$ is a Lipschitz bounded domain and $d_{\Omega^{\prime}} \leq d_{\Omega}$ $\left(d_{\Omega^{\prime}} \leq 3 R d_{\Omega}\right.$ if $\left.\Omega=\mathbb{R}^{2}\right)$. Therefore, we infer from the classical Hardy inequality on bounded Lipschitz domains, see e.g. [22], that for every $u \in \mathcal{D}\left(\Omega^{\prime}\right)$,

$$
\int_{\Omega^{\prime}} \frac{|u|^{2}}{d_{\Omega}(x)^{2}} \leq \int_{\Omega^{\prime}} \frac{|u|^{2}}{d_{\Omega^{\prime}}(x)^{2}} \leq C_{1} \int_{\Omega^{\prime}}|\nabla u|^{2}
$$

where $C_{1}>0$. 
On the other hand, if $|x|>2 R$, then $d_{\Omega}(x) \geq|x| / 2$, so that for every $u \in \mathcal{D}\left(\mathbb{R}^{N} \backslash B(0,2 R)\right)$, we obtain

$$
\int_{\mathbb{R}^{N} \backslash B(0,2 R)} \frac{|u|^{2}}{d_{\Omega}(x)^{2}} \leq 4 \int_{\mathbb{R}^{N} \backslash B(0,2 R)} \frac{|u|^{2}}{|x|^{2}} \leq \frac{4}{c} \int_{\mathbb{R}^{N} \backslash B(0,2 R)} V(x)|u|^{2} .
$$

We next choose a cut-off function $\eta \in \mathcal{D}(\Omega)$ such that $\eta(x)=1$ if $x \in$ $B(0,2 R), 0 \leq \eta(x) \leq 1$ if $x \in B(0,3 R)$ and $\eta(x)=0$ outside $B(0,3 R)$. Combining these inequalities, we then get for every $u \in \mathcal{D}(\Omega)$,

$$
\begin{aligned}
\frac{1}{2} \int_{\Omega} \frac{|u|^{2}}{d_{\Omega}(x)^{2}} & \leq \int_{\Omega \cap B(0,3 R)} \frac{|\eta u|^{2}}{d_{\Omega}(x)^{2}}+\int_{\mathbb{R}^{N} \backslash B(0,2 R)} \frac{|(1-\eta) u|^{2}}{d_{\Omega}(x)^{2}} \\
& \leq C_{1} \int_{\Omega \cap B(0,3 R)}|\nabla(\eta u)|^{2}+\frac{4}{c} \int_{\mathbb{R}^{N} \backslash B(0,2 R)} V(x)|u|^{2} \\
& \leq 2 C_{1} \int_{\Omega}|\nabla u|^{2}+\left(C_{2}\|\nabla \eta\|_{\infty}^{2}+\frac{4}{c}\right) \int_{\Omega} V(x)|u|^{2},
\end{aligned}
$$

where $C_{2}>0$ essentially depends on the lower bound of $V$ in the annulus $B(0,3 R) \backslash B(0,2 R)$. This concludes the proof.

Proof of Lemma 6. Throughout the proof, $C$ denotes a positive constant that can change from line to line. Let $\left(u_{n}\right)_{n} \subset \mathcal{H}$ be a Palais-Smale sequence, that is $\mathcal{J}\left(u_{n}\right)$ is bounded and $\mathcal{J}^{\prime}\left(u_{n}\right) \rightarrow 0$ as $n \rightarrow \infty$.

Claim 1: the sequence $\left(u_{n}\right)_{n}$ is bounded in $\mathcal{H}$. By assumption, we have

$$
\left|\frac{1}{2} \int_{\Omega}\left(\left|\nabla u_{n}\right|^{2}+V(x)\left|u_{n}\right|^{2}\right)-\int_{\Omega} G\left(x, u_{n}\right)\right| \leq C
$$

and for $n$ large enough,

$$
\begin{aligned}
\left|\left(\mathcal{J}^{\prime}\left(u_{n}\right), u_{n}\right)\right| & =\left|\int_{\Omega}\left(\left|\nabla u_{n}\right|^{2}+V(x)\left|u_{n}\right|^{2}\right)-\int_{\Omega} g\left(x, u_{n}\right) u_{n}\right| \\
& \leq\left\|\mathcal{J}^{\prime}\left(u_{n}\right)\right\|\left\|u_{n}\right\| \leq\left\|u_{n}\right\| .
\end{aligned}
$$

Combining these inequalities, we infer from $\left(g_{3}\right)$ that

$$
\frac{\theta-2}{2} \int_{\Omega}\left(\left|\nabla u_{n}\right|^{2}+V(x)\left|u_{n}\right|^{2}\right) \leq \kappa \frac{\theta-2}{2} \int_{\Omega \backslash \Lambda} V(x)\left|u_{n}\right|^{2}+C+\left\|u_{n}\right\| .
$$

Since $\kappa<1$ in (4.1) it follows that

$$
\int_{\Omega}\left(\left|\nabla u_{n}\right|^{2}+V(x)\left|u_{n}\right|^{2}\right) \leq C\left(1+\left\|u_{n}\right\|\right) .
$$

This proves the claim. 
Claim 2: for all $\delta>0$, there exists a compact set $Z \subset \Omega$ such that

$$
\limsup _{n \rightarrow \infty} \int_{\Omega \backslash Z}\left(\left|\nabla u_{n}\right|^{2}+V(x)\left|u_{n}\right|^{2}\right) \leq \delta .
$$

Let $\delta>0$ be given. We define a cut-off function $\eta_{\lambda}$ in the following way. Assume that $\zeta \in C^{\infty}(\mathbb{R}, \mathbb{R})$ is such that $0 \leq \zeta(s) \leq 1$ if $|s| \in[1,2]$ and

$$
\zeta(s)= \begin{cases}0 & \text { if }|s| \leq 1 \\ 1 & \text { if }|s| \geq 2\end{cases}
$$

We then define $\eta_{\lambda} \in C^{\infty}\left(\mathbb{R}^{n}, \mathbb{R}\right)$ by

$$
\eta_{\lambda}(x):=\zeta\left(\frac{\log d_{\Omega}(x)}{\lambda}\right)
$$

where again $d_{\Omega}(x)=d(x, \partial \Omega)$ if $\partial \Omega \neq \emptyset$ while $d_{\Omega}(x)=1+|x|$ when $\Omega=\mathbb{R}^{N}$. Notice that the function $d_{\Omega}$ is Lipschitz and $\left|\nabla d_{\Omega}\right| \leq 1$ almost everywhere so that $\eta_{\lambda} u_{n} \in \mathcal{H}$. Since $\left(u_{n}\right)$ is bounded, we infer that

$$
\left(\mathcal{J}^{\prime}\left(u_{n}\right), \eta_{\lambda} u_{n}\right)=o(1) \quad \text { as } n \rightarrow \infty
$$

Assuming that $\lambda$ has been chosen large enough so that $\eta_{\lambda} \equiv 0$ on $\Lambda$, we then compute

$$
\int_{\Omega}\left(\left|\nabla u_{n}\right|^{2}+V(x)\left|u_{n}\right|^{2}\right) \eta_{\lambda}=\int_{\Omega} g\left(x, u_{n}\right) u_{n} \eta_{\lambda}-\int_{\Omega} u_{n} \nabla u_{n} \cdot \nabla \eta_{\lambda}+o(1) .
$$

For the first term in the right-hand side of the equality, we observe that since $\eta_{\lambda}(x)=0$ for any $x \in \Lambda$, we have

$$
\int_{\Omega} g\left(x, u_{n}\right) u_{n} \eta_{\lambda} \leq \kappa \int_{\Omega} V(x)\left|u_{n}\right|^{2} \eta_{\lambda}
$$

The second term can be rewritten as

$$
\int_{\Omega} u_{n} \nabla u_{n} \cdot \nabla \eta_{\lambda}=\int_{\Omega} \frac{u_{n}}{d_{\Omega}(x)} \nabla u_{n} \cdot\left(d_{\Omega}(x) \nabla \eta_{\lambda}\right) .
$$

From the classical Hardy inequality if $N>2$ or $\Omega$ is bounded (see e.g. [22]), or Lemma 7 when $N=2$ and $\Omega$ is not bounded, one has

$$
\int_{\Omega} \frac{\left|u_{n}\right|^{2}}{d_{\Omega}(x)^{2}} \leq C\left\|u_{n}\right\|_{\mathcal{H}}^{2}
$$


On the other hand, we compute

$$
\left\|d_{\Omega} \nabla \eta_{\lambda}\right\|_{\infty}=\sup _{x \in \Omega}\left|d_{\Omega}(x) \zeta^{\prime}\left(\frac{\log d_{\Omega}(x)}{\lambda}\right) \frac{1}{\lambda d_{\Omega}(x)} \nabla d_{\Omega}(x)\right| \leq \frac{C}{\lambda} .
$$

Combining these last estimates with Cauchy-Schwarz inequality, we infer that

$$
\left|\int_{\Omega} u_{n} \nabla u_{n} \cdot \nabla \eta_{\lambda}\right| \leq \frac{C}{\lambda}\left\|u_{n}\right\|_{\mathcal{H}}^{2}
$$

Now, taking (4.9) and (4.10) into account, we deduce from (4.8) that

$$
(1-\kappa) \int_{\Omega}\left(\left|\nabla u_{n}\right|^{2}+V(x)\left|u_{n}\right|^{2}\right) \eta_{\lambda} \leq \frac{C}{\lambda}\left\|u_{n}\right\|_{\mathcal{H}}+o(1) .
$$

If $\lambda>0$ is sufficiently large, (4.6) thus holds with

$$
Z=\left\{x \in \Omega: \mathrm{e}^{-2 \lambda} \leq d_{\Omega}(x) \leq \mathrm{e}^{2 \lambda}\right\} .
$$

Conclusion. We deduce from Claim 1 that, up to a subsequence, $\left(u_{n}\right)_{n}$ converges weakly in $\mathcal{H}$ to some function $u \in \mathcal{H}$. Now, fix $\delta>0$ and let $Z \supset \Lambda$ be such that (4.6) holds and

$$
\int_{\Omega \backslash Z} V(x)|u|^{2} \leq \delta .
$$

Let us write

$$
\left\|u_{n}-u\right\|_{\mathcal{H}}^{2}=\left(\mathcal{J}^{\prime}\left(u_{n}\right)-\mathcal{J}^{\prime}(u), u_{n}-u\right)+\int_{\Omega}\left(g\left(x, u_{n}\right)-g(x, u)\right)\left(u_{n}-u\right) d x .
$$

Since $\mathcal{J}^{\prime}\left(u_{n}\right) \rightarrow 0$ and $u_{n}$ converges weakly to $u,\left(\mathcal{J}^{\prime}\left(u_{n}\right)-\mathcal{J}^{\prime}(u), u_{n}-u\right) \rightarrow 0$ as $n \rightarrow \infty$. By $\left(g_{3}\right)$, one has

$$
\begin{aligned}
\limsup _{n \rightarrow \infty} \int_{\Omega \backslash Z}\left|g\left(x, u_{n}\right)-g(x, u)\right|\left|u_{n}-u\right| d x & \\
& \leq \limsup _{n \rightarrow \infty} 2 \kappa \int_{\Omega \backslash Z} V(x)\left(\left|u_{n}\right|^{2}+|u|^{2}\right) \leq 4 \kappa \delta,
\end{aligned}
$$

where the second inequality comes from (4.6) and Fatou's Lemma. On the other hand, by Rellich's compactness theorem, $u_{n} \rightarrow u$ in $L^{p+1}(Z)$ so that by $\left(g_{2}\right)$,

$$
\int_{Z}\left(g\left(x, u_{n}\right)-g(x, u)\right)\left(u_{n}-u\right) d x \rightarrow 0 .
$$

One thus concludes that for every $\delta>0$,

$$
\limsup _{n \rightarrow \infty}\left\|u_{n}-u\right\|_{\mathcal{H}}^{2} \leq 4 \kappa \delta
$$

i.e. $u_{n} \rightarrow u$ strongly in $\mathcal{H}$. 


\subsection{Solutions of the modified problem}

Having proved that (PS) holds, we may state the following existence result for the modified problem (4.3).

Proposition 9. Suppose that $g: \mathbb{R}^{+} \rightarrow \mathbb{R}$ is a Carathéodory function satisfying $\left(g_{1}\right)-\left(g_{3}\right)$ and $V \in C(\Omega)$ is positive. If $N=2$ and $\Omega$ is unbounded, assume moreover that $V$ satisfies

$$
\liminf _{|x| \rightarrow \infty} V(x)|x|^{2}>0 .
$$

Then, the functional $\mathcal{J}_{\varepsilon}$ has a critical point $u_{\varepsilon} \in \mathcal{H}_{\varepsilon}$ which is a weak positive solution of (4.3).

The proof being straightforward, we skip it. We next analyze the regularity of the solution.

Proposition 10. Under the assumptions of Proposition 9, any solution $u_{\varepsilon} \in \mathcal{H}_{\varepsilon}$ of (4.3) satisfies $u_{\varepsilon} \in W_{\text {loc }}^{2, q}(\Omega)$ for every $q<\infty$. In particular, $u \in C_{\mathrm{loc}}^{1, \alpha}(\Omega)$ for every $0<\alpha<1$.

The proof follows from a classical bootstrap argument so that we omit it.

Remark 11. Observe that this result cannot be improved, even if we add further regularity assumptions on $\Omega$ and $V$. Indeed, in general, one cannot prove that $u \in C^{2, \alpha}(\Omega)$ or $u \in W_{\mathrm{loc}}^{3,1}(\Omega)$. This is due to the fact that even for a smooth $u, g(x, u)$ does not need to be neither in $C^{0, \alpha}(\Omega)$ nor in $W_{\mathrm{loc}}^{1,1}(\Omega)$.

\subsection{Estimate of the moutain pass level}

We deduce from the preceding sections that the mountain pass level $c_{\varepsilon}$ defined by (4.4) is a critical level for $\mathcal{J}_{\varepsilon}$. In order to prove that this minimax level yields a solution of the original problem for small values of $\varepsilon$, we need a sharp energy estimate.

Let $\Lambda \subset \subset \Omega$ be such that

$$
\inf _{\xi \in \Lambda} \mathcal{C}(\xi)<\inf _{\xi \in \partial \Lambda} \mathcal{C}(\xi)
$$

and $\sup _{\xi \in \Lambda} \mathcal{C}(\xi)<\infty$. As already mentioned, the continuity of $V$ and $K$ implies that the concentration function $\mathcal{C}$ is continuous in $\Lambda$. We therefore deduce the existence of $\xi_{0} \in \Lambda$ such that

$$
\mathcal{C}\left(\xi_{0}\right)=\min _{\Lambda} \mathcal{C}
$$


To save notation, let us denote by $\mathcal{F}_{0}: H^{1}\left(\mathbb{R}^{N}\right) \rightarrow \mathbb{R}$ the functional defined by (3.1) with $a=V\left(\xi_{0}\right)$ and $b=K\left(\xi_{0}\right)$. We also define

$$
c_{0}:=\inf _{\gamma \in \Gamma_{0}} \max _{t \in[0,1]} \mathcal{F}_{0}(\gamma(t)),
$$

where

$$
\Gamma_{0}:=\left\{\gamma \in C\left([0,1], H^{1}\left(\mathbb{R}^{N}\right)\right) \mid \gamma(0)=0, \mathcal{F}_{0}(\gamma(1))<0\right\} .
$$

One of the key ingredients to prove Theorem 1 is a comparison between the levels $c_{\varepsilon}$ and $c_{0}$ for $\varepsilon$ small.

Lemma 12. Suppose that $f: \mathbb{R}^{+} \rightarrow \mathbb{R}^{+}$is a continuous function satisfying $\left(f_{1}\right)-\left(f_{3}\right), V, K: \Omega \rightarrow \mathbb{R}^{+}$are continuous functions and $g: \Omega \times \mathbb{R}^{+} \rightarrow \mathbb{R}$ is defined by (4.2). If $N=2$ and $\Omega$ is unbounded, assume moreover that $V$ satisfies

$$
\liminf _{|x| \rightarrow \infty} V(x)|x|^{2}>0 .
$$

Then, the functional $\mathcal{J}_{\varepsilon}$ has a critical point $u_{\varepsilon} \in \mathcal{H}_{\varepsilon}$ such that

$$
\mathcal{J}_{\varepsilon}\left(u_{\varepsilon}\right) \leq \varepsilon^{N} c_{0}+o\left(\varepsilon^{N}\right), \quad \text { as } \varepsilon \rightarrow 0 .
$$

Moreover, there exists $C>0$ such that

$$
\left\|u_{\varepsilon}\right\|_{\mathcal{H}_{\varepsilon}}^{2} \leq C \varepsilon^{N}
$$

Proof. It follows from Proposition 9 that the mountain pass value $c_{\varepsilon}$ is a critical level for $\mathcal{J}_{\varepsilon}$. From the definition of $c_{0}$, we infer that for every $\delta>0$, there exists a continuous path $\gamma_{\delta}:[0,1] \rightarrow H^{1}\left(\mathbb{R}^{N}\right)$ such that $\gamma_{\delta}(0)=0$, $\mathcal{F}_{0}\left(\gamma_{\delta}(1)\right)<0$ and

$$
c_{0} \leq \max _{t \in[0,1]} \mathcal{F}_{0}\left(\gamma_{\delta}(t)\right) \leq c_{0}+\delta .
$$

Let $\eta \in C^{\infty}\left(\mathbb{R}^{N}, \mathbb{R}\right)$ be a cut-off function, with support in $\Lambda$, such that $\eta(x)=1$ for $x$ in a neighbourhood of $\xi_{0}$ defined by (4.12). We then define the path $\bar{\gamma}_{\delta}:[0,1] \rightarrow \mathcal{H}_{\varepsilon}$ by

$$
\bar{\gamma}_{\delta}(t): x \rightarrow \eta(x) \gamma_{\delta}(t)\left(\frac{x-\xi_{0}}{\varepsilon}\right) .
$$

Rescaling in the space variable leads to

$$
\begin{aligned}
\mathcal{J}_{\varepsilon}\left(\bar{\gamma}_{\delta}(t)\right)= & \frac{\varepsilon^{N}}{2} \int_{\Omega_{\varepsilon}}\left(\left|\nabla\left(\eta\left(\varepsilon x+\xi_{0}\right) \gamma_{\delta}(t)\right)\right|^{2}+V\left(\varepsilon x+\xi_{0}\right)\left|\eta\left(\varepsilon x+\xi_{0}\right) \gamma_{\delta}(t)\right|^{2}\right) \\
& -\varepsilon^{N} \int_{\Omega_{\varepsilon}} K\left(\varepsilon x+\xi_{0}\right) F\left(\eta\left(\varepsilon x+\xi_{0}\right) \gamma_{\delta}(t)\right),
\end{aligned}
$$


where $\Omega_{\varepsilon}:=\left\{x \in \mathbb{R}^{N} \mid \varepsilon x+\xi_{0} \in \Omega\right\}$. Hence, a straightforward but rather tedious computation shows that

$$
\mathcal{J}_{\varepsilon}\left(\bar{\gamma}_{\delta}(t)\right)=\varepsilon^{N} \mathcal{F}_{0}\left(\gamma_{\delta}(t)\right)+o\left(\varepsilon^{N}\right) \text { as } \varepsilon \rightarrow 0
$$

It follows that for $\varepsilon$ small enough, $\bar{\gamma}_{\delta}$ belongs to the class of paths $\Gamma_{\varepsilon}$ defined by (4.5). We therefore conclude that

$$
\begin{aligned}
\mathcal{J}_{\varepsilon}\left(u_{\varepsilon}\right) & =\inf _{\gamma \in \Gamma_{\varepsilon}} \max _{t \in[0,1]} \mathcal{J}_{\varepsilon}(\gamma(t)) \\
& \leq \max _{t \in[0,1]} \mathcal{J}_{\varepsilon}\left(\bar{\gamma}_{\delta}(t)\right) \\
& \leq \varepsilon^{N}\left(c_{0}+\delta\right)+o\left(\varepsilon^{N}\right) .
\end{aligned}
$$

Since the last inequality holds for any $\delta>0$, the first statement is established.

Consider now a critical point $u_{\varepsilon} \in \mathcal{H}_{\varepsilon}$ at the mountain pass energy level. To prove the estimate on the norm of $u_{\varepsilon}$, we observe, arguing as in the first claim of Lemma 6 , that

$$
(1-\kappa)\left(\frac{1}{2}-\frac{1}{\theta}\right) \int_{\Omega}\left(\varepsilon^{2}\left|\nabla u_{\varepsilon}\right|^{2}+V(x)\left|u_{\varepsilon}\right|^{2}\right) \leq \mathcal{J}_{\varepsilon}\left(u_{\varepsilon}\right) .
$$

Hence the proof follows from the energy estimate.

\section{Asymptotics of solutions}

We study in this section, the asymptotic behaviour of the mountain pass solution of the modified problem (4.3) as $\varepsilon \rightarrow 0$. In particular, we derive a uniform estimate of $u_{\varepsilon}$ on $\partial \Lambda$, see Proposition 21, which is a main step in the proof of the existence of a solution of the original boundary value problem (2.1).

\subsection{Estimates on sequences of rescaled solutions}

Since the $\mathcal{H}_{\varepsilon}$-norm of the solution $u_{\varepsilon}$ of the modified problem is of the order $\varepsilon^{N / 2}$, it is natural to rescale $u_{\varepsilon}$ as $u_{\varepsilon}\left(x_{\varepsilon}+\varepsilon \cdot\right)$ around a well-chosen family of points $x_{\varepsilon}$.

We first observe that such sequences are relatively compact for the uniform $C^{1}$-convergence over compact sets. Moreover, even if there is no a priori guarantee that $u_{\varepsilon}$ belongs to $H^{1}(\Omega)$, any limit $v$ of a subsequence of rescaled solutions will be in $H^{1}\left(\mathbb{R}^{N}\right)$, i.e. the fact that $u_{\varepsilon} \in H_{\text {loc }}^{1}\left(\mathbb{R}^{N}\right)$ yields $v \in H^{1}\left(\mathbb{R}^{N}\right)$. 
Lemma 13. Suppose the assumptions of Lemma 12 are satisfied and assume $u_{\varepsilon} \in H_{\mathrm{loc}}^{1}(\Omega)$ is the positive solution of (4.3) obtained in that lemma. Let $\left(\varepsilon_{n}\right)_{n} \subset \mathbb{R}^{+}$and $\left(x_{n}\right)_{n} \subset \Omega$ be sequences such that $\varepsilon_{n} \rightarrow 0$ and $x_{n} \rightarrow \bar{x} \in \Omega$ as $n \rightarrow \infty$ and denote by $\left(v_{n}\right)_{n}$ the sequence defined by $v_{n}(x)=u_{\varepsilon_{n}}\left(x_{n}+\varepsilon_{n} x\right)$. Then, there exists $v \in H^{1}\left(\mathbb{R}^{N}\right)$ such that, along a subsequence that we still denote by $\left(v_{n}\right)_{n}$,

$$
v_{n} \stackrel{C_{\text {loc }}^{1}\left(\mathbb{R}^{N}\right)}{\longrightarrow} v .
$$

Moreover, for every $R>0$ and $q>0$, we have

$$
\sup _{n \in \mathbb{N}}\left\|v_{n}\right\|_{W^{2, q}\left(B_{R}\right)}<\infty
$$

and

$$
\begin{aligned}
\int_{\mathbb{R}^{N}}|\nabla v|^{2} & =\lim _{R \rightarrow \infty} \lim _{n \rightarrow \infty} \varepsilon_{n}^{-N} \int_{B_{n}(R)} \varepsilon_{n}^{2}\left|\nabla u_{\varepsilon_{n}}\right|^{2}, \\
\int_{\mathbb{R}^{N}} V(\bar{x})|v|^{2} & =\lim _{R \rightarrow \infty} \lim _{n \rightarrow \infty} \varepsilon_{n}^{-N} \int_{B_{n}(R)} V(x)\left|u_{\varepsilon_{n}}\right|^{2},
\end{aligned}
$$

where $B_{n}(R):=B\left(x_{n}, \varepsilon_{n} R\right)$. In particular, we have

$$
\lim _{R \rightarrow \infty} \lim _{n \rightarrow \infty} \varepsilon_{n}^{-N} \int_{A_{n}(R)} \varepsilon_{n}^{2}\left|\nabla u_{\varepsilon_{n}}\right|^{2}+V(x)\left|u_{\varepsilon_{n}}\right|^{2}=0,
$$

where $A_{n}(R):=B\left(x_{n}, \varepsilon_{n} R\right) \backslash B\left(x_{n}, \varepsilon_{n} R / 2\right)$.

Proof. First observe that each $v_{n}$ solves the equation

$$
\begin{aligned}
-\Delta v_{n}+V\left(x_{n}+\varepsilon_{n} x\right) & v_{n}=\chi_{\Lambda}\left(x_{n}+\varepsilon_{n} x\right) K\left(x_{n}+\varepsilon_{n} x\right) f\left(v_{n}\right) \\
& +\left(1-\chi_{\Lambda}\left(x_{n}+\varepsilon_{n} x\right)\right) \tilde{f}\left(x_{n}+\varepsilon_{n} x, v_{n}\right), x \in \Omega_{n},
\end{aligned}
$$

where $\Omega_{n}:=\left\{x \in \mathbb{R}^{N} \mid x_{n}+\varepsilon_{n} x \in \Omega\right\}$. We now infer from the estimates of Lemma 12 that

$$
\mathcal{J}_{\varepsilon_{n}}\left(v_{n}\right) \leq c_{0}+o(1) \text { as } n \rightarrow \infty
$$

and for all $n \in \mathbb{N}$,

$$
\int_{\Omega_{n}}\left(\left|\nabla v_{n}\right|^{2}+V\left(x_{n}+\varepsilon_{n} x\right)\left|v_{n}\right|^{2}\right)<C
$$

with $C>0$ independent of $n$.

Define again a cut-off function $\eta_{R} \in C_{c}^{\infty}\left(\mathbb{R}^{N}\right)$ such that $\eta(x)=1$ if $|x| \leq R / 2, \eta(x)=0$ if $|x| \geq R$ and $\left\|\nabla \eta_{R}\right\|_{\infty} \leq C / R$ for some $C>0$. Choose $R_{n}$ such that $R_{n} \rightarrow \infty, \varepsilon_{n} R_{n} \rightarrow 0$ and $B\left(x_{n}, 2 \varepsilon_{n} R_{n}\right) \subset \Omega$ and define $w_{n} \in H_{\mathrm{loc}}^{1}\left(\mathbb{R}^{N}\right)$ by

$$
w_{n}(x):=\eta_{R_{n}}(x) v_{n}(x) .
$$


We first observe that

$$
\int_{\mathbb{R}^{N}}\left|w_{n}\right|^{2} \leq \int_{B_{R_{n}}}\left|v_{n}\right|^{2} \leq \frac{1}{\inf _{B_{n}(R)} V} \int_{\Omega_{n}} V\left(x_{n}+\varepsilon_{n} x\right)\left|v_{n}\right|^{2} .
$$

Observe that $V$ being positive in $\Omega$, the convergence of $x_{n}$ to a point in $\Omega$ implies there exists $m>0$ such that $V(x) \geq m$ for every $x \in B\left(x_{n}, \varepsilon_{n} R_{n}\right)$. Hence we deduce from (5.5) the estimate

$$
\int_{\mathbb{R}^{N}}\left|w_{n}\right|^{2} \leq \frac{C}{m}
$$

On the other hand, we compute

$$
\int_{\mathbb{R}^{N}}\left|\nabla w_{n}\right|^{2}=\int_{\mathbb{R}^{N}}\left|\nabla \eta_{R_{n}}\right|^{2}\left|v_{n}\right|^{2}+\int_{\mathbb{R}^{N}}\left|\nabla v_{n}\right|^{2}\left|\eta_{R_{n}}\right|^{2}+2 \int_{\mathbb{R}^{N}} \nabla v_{n} \cdot \nabla \eta_{R_{n}} \eta_{R_{n}} v_{n} .
$$

For the first term on the right-hand side, we have the estimate

$$
\left.\left|\int_{\mathbb{R}^{N}}\right| \nabla \eta_{R_{n}}\right|^{2}\left|v_{n}\right|^{2} \mid \leq \frac{C}{R_{n}}\left\|v_{n}\right\|_{L^{2}\left(B_{R_{n}}\right)},
$$

while for the last one, we infer from Cauchy-Schwarz inequality that

$$
\left|\int_{\mathbb{R}^{N}} \nabla v_{n} \cdot \nabla \eta_{R_{n}} \eta_{R_{n}} v_{n}\right| \leq \frac{C}{R_{n}}\left\|\nabla v_{n}\right\|_{L^{2}\left(B_{R_{n}}\right)}\left\|v_{n}\right\|_{L^{2}\left(B_{R_{n}}\right)} .
$$

Since we have

$$
\left\|v_{n}\right\|_{H^{1}\left(B_{R_{n}}\right)} \leq \frac{1}{m} \int_{\Omega_{n}}\left(\left|\nabla v_{n}\right|^{2}+V\left(x_{n}+\varepsilon_{n} x\right)\left|v_{n}\right|^{2}\right) \leq \frac{C}{m},
$$

collecting the estimates (5.6), (5.7), (5.8) and (5.9), we infer that $\left(w_{n}\right)_{n}$ is bounded in $H^{1}\left(\mathbb{R}^{N}\right)$. Since $w_{n}$ solves $(5.3)$ on $B_{R_{n} / 2}$ for all $n$, classical regularity estimates yield then (5.1).

We may now extract from $\left(w_{n}\right)_{n}$ a subsequence, that we still denote by $\left(w_{n}\right)_{n}$ for simplicity, that converges weakly in $H^{1}\left(\mathbb{R}^{N}\right)$ to some function $v \in H^{1}\left(\mathbb{R}^{N}\right)$. By (5.1), it is clear that $w_{n}$ converges to $v$ uniformly in $C^{1}(K)$, for every compact $K \subset \mathbb{R}^{N}$. Moreover, for $n$ large enough, $w_{n}=v_{n}$ in compact sets so that $v_{n} \rightarrow v$ in $C_{\text {loc }}^{1}\left(\mathbb{R}^{N}\right)$.

The remaining estimates follow from the continuity of $V$ and the fact that $v \in H^{1}\left(\mathbb{R}^{N}\right)$.

A useful application of Lemma 13 consists in estimating the action of $u_{\varepsilon}$ in neighbourhoods of points. In particular, this will provide a lower bound of the action depending on the number and on the location of the local maxima of $u_{\varepsilon}$. 
Lemma 14. Suppose that the assumptions of Lemma 12 are satisfied and assume moreover that $\left(f_{4}\right)$ holds. Let $u_{\varepsilon} \in H_{\mathrm{loc}}^{1}(\Omega)$ be the positive solution of (4.3) obtained in Lemma 12 and the sequences $\left(\varepsilon_{n}\right)_{n} \subset \mathbb{R}^{+}$and $\left(x_{n}\right)_{n} \subset \Omega$ be such that $\varepsilon_{n} \rightarrow 0, x_{n} \rightarrow \bar{x}$ as $n \rightarrow \infty$ and

$$
\liminf _{n \rightarrow \infty} u_{\varepsilon_{n}}\left(x_{n}\right)>0
$$

Then, up to a subsequence, we have

$$
\liminf _{R \rightarrow \infty} \liminf _{n \rightarrow \infty} \varepsilon_{n}^{-N}\left(\int_{B_{n}(R)} \frac{1}{2}\left(\varepsilon_{n}^{2}\left|\nabla u_{\varepsilon_{n}}\right|^{2}+V(x)\left|u_{\varepsilon_{n}}\right|^{2}\right)-G\left(x, u_{\varepsilon_{n}}\right)\right) \geq \mathcal{C}(\bar{x}),
$$

where $B_{n}(R):=B\left(x_{n}, \varepsilon_{n} R\right)$.

Proof. Passing to a subsequence if necessary, we may assume that there exists $v \in H^{1}\left(\mathbb{R}^{N}\right)$ such if $v_{n}(y)=u_{\varepsilon_{n}}\left(x_{n}+\varepsilon_{n} y\right), v_{n} \rightarrow v$ in $C_{\text {loc }}^{1}\left(\mathbb{R}^{N}\right)$. Since $\Lambda$ is smooth, still going to a subsequence if required, the sequence of characteristic functions $\chi_{n}(x)=\chi_{\Lambda}\left(x_{n}+\varepsilon_{n} x\right)$ converges almost everywhere to a measurable function $\chi$ satisfying $0 \leq \chi(x) \leq 1$. We then deduce that $v$ solves the limiting equation

$$
-\Delta v+V(\bar{x}) v=\tilde{g}(x, v), \quad x \in \mathbb{R}^{N},
$$

where

$$
\tilde{g}(x, v):=\chi(x) K(\bar{x}) f(v)+(1-\chi(x)) \tilde{f}(\bar{x}, v) .
$$

By (5.10), we know that $v(0)=\lim _{n \rightarrow \infty} v_{n}(0)>0$, so that $v$ is not identically zero.

As $v$ is a nonzero solution of (5.12), it belongs to the Nehari manifold associated to this equation, that is

$$
\mathcal{N}:=\left\{u \in H^{1}\left(\mathbb{R}^{N}\right) \mid u \neq 0,\left(\mathcal{G}^{\prime}(u), u\right)=0\right\},
$$

$\mathcal{G}: H^{1}\left(\mathbb{R}^{N}\right) \rightarrow \mathbb{R}$ being the functional defined by

$$
\mathcal{G}(u):=\frac{1}{2}\left(\int_{\mathbb{R}^{N}}|\nabla u|^{2}+V(\bar{x}) \int_{\mathbb{R}^{N}}|u|^{2}\right)-\int_{\mathbb{R}^{N}} \tilde{G}(x, u),
$$

where

$$
\tilde{G}(x, u):=\int_{0}^{u} \tilde{g}(x, s) d s .
$$

Since $\tilde{g}(x, v) \leq K(\bar{x}) f(v)$ in $\mathbb{R}^{N} \times \mathbb{R}^{+}$, it follows that for all $u \in H^{1}\left(\mathbb{R}^{N}\right)$,

$$
\mathcal{G}(u) \geq \frac{1}{2}\left(\int_{\mathbb{R}^{N}}|\nabla u|^{2}+V(\bar{x}) \int_{\mathbb{R}^{N}}|u|^{2}\right)-K(\bar{x}) \int_{\mathbb{R}^{N}} F(u) .
$$


Therefore, as $\tilde{g}$ satisfies the condition $\left(g_{4}\right)$, we deduce that

$$
\begin{aligned}
\mathcal{G}(v)=\max _{t>0} \mathcal{G}(t v) & \geq \inf _{\substack{u \in H^{1}\left(\mathbb{R}^{N}\right) \\
u \neq 0}} \sup _{t>0} \mathcal{G}(t u) \\
& \geq \inf _{\substack{u \in H^{1}\left(\mathbb{R}^{N}\right) \\
u \neq 0}} \sup _{t>0} \mathcal{F}_{V(\bar{x}), K(\bar{x})}(t u)=\mathcal{C}(\bar{x}) .
\end{aligned}
$$

Finally, we claim that

$$
\liminf _{R \rightarrow \infty} \liminf _{n \rightarrow \infty} \frac{1}{2} \int_{B_{R}}\left|\nabla v_{n}\right|^{2}+V(x)\left|v_{n}\right|^{2}-\int_{B_{R}} G\left(x, v_{n}\right) \geq \mathcal{G}(v) .
$$

Let us write for notational convenience

$$
h_{n}:=\frac{1}{2}\left(\left|\nabla v_{n}\right|^{2}+V\left(x_{n}+\varepsilon_{n} x\right)\left|v_{n}\right|^{2}\right)-G\left(x_{n}+\varepsilon_{n} x, v_{n}\right) .
$$

Then, for every $R>0$, the convergence of $v_{n}$ in $C_{\text {loc }}^{1}\left(\mathbb{R}^{N}\right)$ implies that

$$
\lim _{n \rightarrow \infty} \int_{B_{R}} h_{n}=\frac{1}{2} \int_{B_{R}}\left(|\nabla v|^{2}+V(\bar{x})|v|^{2}\right)-\int_{B_{R}} \tilde{G}(x, v) .
$$

On the other hand, since $v \in H^{1}\left(\mathbb{R}^{N}\right)$, for any $\delta>0$, there exists $R_{0}>0$ such that if $R>R_{0}$

$$
\lim _{n \rightarrow \infty} \int_{B_{R}} h_{n} \geq \mathcal{G}(v)-\delta
$$

This proves the claim and completes the proof.

The estimate (5.11) only becomes useful once we can estimate what happens outside small balls. That is the object of the next lemma.

Lemma 15. Let the assumptions of Lemma 12 be satisfied and assume $u_{\varepsilon} \in$ $H_{\text {loc }}^{1}(\Omega)$ is the positive solution of (4.3) obtained in that lemma. Let $\left(\varepsilon_{n}\right)_{n} \subset$ $\mathbb{R}^{+}$and $\left(x_{n}^{i}\right)_{n} \subset \Omega, 1 \leq i \leq K$, be sequences such that $\varepsilon_{n} \rightarrow 0$ and $x_{n}^{i} \rightarrow$ $\bar{x}^{i} \in \bar{\Lambda}$ as $n \rightarrow \infty$. Then, up to a subsequence, we have

$$
\begin{aligned}
& \liminf _{R \rightarrow \infty} \liminf _{n \rightarrow \infty} \varepsilon_{n}^{-N}\left(\int_{\Omega \backslash \mathcal{B}_{n}(R)} \frac{1}{2}\left(\varepsilon_{n}^{2}\left|\nabla u_{\varepsilon_{n}}\right|^{2}+V(x)\left|u_{\varepsilon_{n}}\right|^{2}\right)-G\left(x, u_{\varepsilon_{n}}\right)\right) \geq 0, \\
& \text { where } \mathcal{B}_{n}(R):=\bigcup_{i=1}^{K} B\left(x_{n}^{i}, \varepsilon_{n} R\right) .
\end{aligned}
$$


Proof. Let $\eta_{R, \varepsilon_{n}}$ be a smooth cut-off function such that $\eta_{R, \varepsilon_{n}} \equiv 0$ in $\mathcal{B}_{n}(R / 2), \eta_{R, \varepsilon_{n}} \equiv 1$ in $\Omega \backslash \mathcal{B}_{n}(R)$ and $\left|\nabla \eta_{R, \varepsilon_{n}}\right| \leq 2 /\left(\varepsilon_{n} R\right)$. As $\left(g_{3}\right)$ holds, we have

$$
\begin{aligned}
\frac{1}{2} \int_{\Omega \backslash \mathcal{B}_{n}(R)} \varepsilon_{n}^{2} \mid \nabla & \left.u_{\varepsilon_{n}}\right|^{2}+V(x)\left|u_{\varepsilon_{n}}\right|^{2}-\int_{\Omega \backslash \mathcal{B}_{n}(R)} G\left(x, u_{\varepsilon_{n}}\right) \\
& \geq \frac{1}{2} \int_{\Omega \backslash \mathcal{B}_{n}(R)} \varepsilon_{n}^{2}\left|\nabla u_{\varepsilon_{n}}\right|^{2}+V(x)\left|u_{\varepsilon_{n}}\right|^{2}-g\left(x, u_{\varepsilon_{n}}\right) u_{\varepsilon_{n}}
\end{aligned}
$$

Taking $u_{\varepsilon_{n}} \eta_{R, \varepsilon_{n}}$ as test function, one obtains

$$
\int_{\Omega} \varepsilon_{n}^{2} \nabla u_{\varepsilon_{n}} \cdot \nabla\left(\eta_{R, \varepsilon_{n}} u_{\varepsilon_{n}}\right)+\eta_{R, \varepsilon_{n}} V(x)\left|u_{\varepsilon_{n}}\right|^{2}-\eta_{R, \varepsilon_{n}} g\left(x, u_{\varepsilon_{n}}\right) u_{\varepsilon_{n}}=0 .
$$

Hence, the right-hand side of (5.14) can be written as

$-\frac{1}{2} \int_{\mathcal{A}_{n}(R)} \varepsilon_{n}^{2} u_{\varepsilon_{n}} \nabla u_{\varepsilon_{n}} \cdot \nabla \eta_{R, \varepsilon_{n}}+\left(\varepsilon_{n}^{2}\left|\nabla u_{\varepsilon_{n}}\right|^{2}+V(x)\left|u_{\varepsilon_{n}}\right|^{2}-g\left(x, u_{\varepsilon_{n}}\right) u_{\varepsilon_{n}}\right) \eta_{R, \varepsilon_{n}}$

where $\mathcal{A}_{n}(R):=\mathcal{B}_{n}(R) \backslash \mathcal{B}_{n}(R / 2)$. For the first term in this expression, using the estimate of Lemma 12 , we infer that

$$
\left|\liminf _{n \rightarrow \infty} \varepsilon_{n}^{-N} \int_{\mathcal{A}_{n}(R)} \varepsilon_{n}^{2} u_{\varepsilon_{n}} \nabla u_{\varepsilon_{n}} \cdot \nabla \eta_{R, \varepsilon_{n}}\right| \leq \frac{C}{R} \sup _{1 \leq i \leq K} \frac{1}{V\left(\bar{x}_{i}\right)^{1 / 2}} .
$$

For the second one, using the growth assumptions on $g$, we get

$$
\begin{array}{r}
\left|\liminf _{n \rightarrow \infty} \varepsilon_{n}^{-N} \int_{\mathcal{A}_{n}(R)}\left(\varepsilon_{n}^{2}\left|\nabla u_{\varepsilon_{n}}\right|^{2}+V(x)\left|u_{\varepsilon_{n}}\right|^{2}-g\left(x, u_{\varepsilon_{n}}\right) u_{\varepsilon_{n}}\right) \eta_{R, \varepsilon_{n}}\right| \\
\leq \liminf _{n \rightarrow \infty} \varepsilon_{n}^{-N} \sum_{i=1}^{K} C_{i}\left(I_{i, n, R}^{2}+I_{i, n, R}^{p+1}\right),
\end{array}
$$

where the constants $C_{i}$ only depend on $\bar{x}_{i}$, and

$$
I_{i, n, R}=\left(\int_{B\left(x_{n}^{i}, \varepsilon_{n} R\right) \backslash B\left(x_{n}^{i}, \varepsilon_{n} R / 2\right)}\left(\varepsilon_{n}^{2}\left|\nabla u_{\varepsilon_{n}}\right|^{2}+V(x)\left|u_{\varepsilon_{n}}\right|^{2}\right) \eta_{R, \varepsilon_{n}} d x\right)^{1 / 2} .
$$

Hence, taking the estimates (5.14) and (5.15) into account, the conclusion follows from Lemma 13.

Proposition 16. Suppose that the assumptions of Lemma 12 are satisfied and assume moreover that $\left(f_{4}\right)$ holds. Let $u_{\varepsilon} \in H_{\mathrm{loc}}^{1}(\Omega)$ be the positive solution of (4.3) obtained in Lemma $12,\left(\varepsilon_{n}\right)_{n} \subset \mathbb{R}^{+}$and $\left(x_{n}^{i}\right)_{n} \subset \Omega$ be sequences 
such that $\varepsilon_{n} \rightarrow 0$ and for $1 \leq i \leq K, x_{n}^{i} \rightarrow \bar{x}^{i} \in \bar{\Lambda}$ as $n \rightarrow \infty$. If for every $1 \leq i<j \leq K$, we have

$$
\limsup _{n \rightarrow \infty} \frac{\left|x_{n}^{i}-x_{n}^{j}\right|}{\varepsilon_{n}}=\infty
$$

and if for every $1 \leq i \leq K$,

$$
\liminf _{n \rightarrow \infty} u_{\varepsilon_{n}}\left(x_{n}^{i}\right)>0
$$

then

$$
\liminf _{n \rightarrow \infty} \varepsilon_{n}^{-N} \mathcal{J}\left(u_{\varepsilon_{n}}\right) \geq \sum_{i=1}^{K} \mathcal{C}\left(\bar{x}_{i}\right)
$$

Proof. First observe that going to a subsequence if necessary, we may assume that for every $1 \leq i<j \leq K$, we have

$$
\lim _{n \rightarrow \infty} \frac{\left|x_{n}^{i}-x_{n}^{j}\right|}{\varepsilon_{n}}=\infty .
$$

We infer from Lemma 14 and Lemma 15 that up to a subsequence, for any fixed $\delta>0$, we can choose $R$ large enough so that

$$
\liminf _{n \rightarrow \infty} \varepsilon_{n}^{-N}\left(\frac{1}{2} \int_{\Omega \backslash \mathcal{B}_{n}(R)} \varepsilon_{n}^{2}\left|\nabla u_{\varepsilon_{n}}\right|^{2}+V(x)\left|u_{\varepsilon_{n}}\right|^{2}-\int_{\Omega \backslash \mathcal{B}_{n}(R)} G\left(x, u_{\varepsilon_{n}}\right)\right) \geq-\delta,
$$

where $\mathcal{B}_{n}(R):=\bigcup_{i=1}^{K} B\left(x_{n}^{i}, \varepsilon_{n} R\right)$ and for every $1 \leq i \leq K$

$\liminf _{n \rightarrow \infty} \varepsilon_{n}^{-N}\left(\frac{1}{2} \int_{B_{n}^{i}(R)} \varepsilon_{n}^{2}\left|\nabla u_{\varepsilon_{n}}\right|^{2}+V(x)\left|u_{\varepsilon_{n}}\right|^{2}-\int_{B_{n}^{i}(R)} G\left(x, u_{\varepsilon_{n}}\right)\right) \geq \mathcal{C}\left(\bar{x}^{i}\right)-\delta$

where $B_{n}^{i}(R):=B\left(x_{n}^{i}, \varepsilon_{n} R\right)$. Now, as for $n$ sufficiently large, the balls $B_{n}^{i}(R)$ are mutually disjoint, we may decompose $\mathcal{J}\left(u_{\varepsilon_{n}}\right)$ as

$$
\begin{aligned}
\mathcal{J}\left(u_{\varepsilon_{n}}\right)= & \sum_{i=1}^{K}\left(\frac{1}{2} \int_{B_{n}^{i}(R)} \varepsilon_{n}^{2}\left|\nabla u_{\varepsilon_{n}}\right|^{2}+V(x)\left|u_{\varepsilon_{n}}\right|^{2}-\int_{B_{n}^{i}(R)} G\left(x, u_{\varepsilon_{n}}\right)\right) \\
& +\frac{1}{2} \int_{\Omega \backslash \cup_{i=1}^{K} B_{n}^{i}(R)} \varepsilon_{n}^{2}\left|\nabla u_{\varepsilon_{n}}\right|^{2}+V(x)\left|u_{\varepsilon_{n}}\right|^{2}-\int_{\Omega \backslash \cup_{i=1}^{K} B_{n}^{i}(R)} G\left(x, u_{\varepsilon_{n}}\right),
\end{aligned}
$$

concluding therefore that

$$
\liminf _{n \rightarrow \infty} \varepsilon_{n}^{-N} \mathcal{J}\left(u_{\varepsilon_{n}}\right) \geq \sum_{i=1}^{K} \mathcal{C}\left(\bar{x}_{i}\right)-(K+1) \delta .
$$

Since this can be done for every subsequence, the conclusion holds for the whole sequence. 
In the case where the $x_{n}$ 's are local maxima of $u_{\varepsilon_{n}}$ and their cluster points are all inside $\Lambda$, the estimates of Lemma 13 can be refined. In particular, we obtain a common decay estimate for any converging subsequence, see Proposition 20. We first consider the following preliminary lemma.

Lemma 17. Let $f \in C(\mathbb{R}), V \in C\left(\Omega ; \mathbb{R}^{+}\right)$and $K \in C\left(\Omega ; \mathbb{R}^{+}\right)$be given and assume $g: \Omega \times \mathbb{R} \rightarrow \mathbb{R}$ is defined by (4.2). Let $u_{\varepsilon} \in H_{\text {loc }}^{1}(\Omega)$ be a positive continuous solution of (4.3). If $y_{\varepsilon} \in \Omega$ is a local maximum point of $u_{\varepsilon}$ such that $u_{\varepsilon}\left(y_{\varepsilon}\right)>0$, then

$$
f\left(u_{\varepsilon}\left(y_{\varepsilon}\right)\right) / u_{\varepsilon}\left(y_{\varepsilon}\right) \geq V\left(y_{\varepsilon}\right) / K\left(y_{\varepsilon}\right) .
$$

Proof. Suppose for the sake of contradiction that (5.16) does not hold. Since $u_{\varepsilon}, V$ and $K$ are continuous, there exists $\rho>0$ such that for every $x \in B\left(y_{\varepsilon}, \rho\right)$, we have

$$
f\left(u_{\varepsilon}(x)\right) / u_{\varepsilon}(x)<V(x) / K(x) .
$$

By definition of $g$, the function $u_{\varepsilon}$ then satisfies the inequality

$$
-\varepsilon^{2} \Delta u_{\varepsilon}+V(x) u_{\varepsilon}<K(x) f\left(u_{\varepsilon}\right)
$$

in $B\left(y_{\varepsilon}, \rho\right)$. Consequently, there holds

$$
-\Delta u_{\varepsilon}<0
$$

in $B\left(y_{\varepsilon}, \rho\right)$. But, we then deduce, using the strong maximum principle for subharmonic functions, that $y_{\varepsilon}$ is not a local maximum of $u_{\varepsilon}$, which is a contradiction.

Proposition 18. Suppose the assumptions of Lemma 12 are satisfied and assume $u_{\varepsilon} \in H_{\mathrm{loc}}^{1}(\Omega)$ is the positive solution of (4.3) obtained in that lemma. Let $\left(\varepsilon_{n}\right)_{n} \subset \mathbb{R}^{+}$and $\left(x_{n}\right)_{n} \subset \Omega$ be sequences such that $\varepsilon_{n} \rightarrow 0, x_{n}$ is a local maximum point of $u_{\varepsilon_{n}}$ and $x_{n} \rightarrow \bar{x} \in \Lambda$ as $n \rightarrow \infty$. Let $\left(v_{n}\right)_{n}$ denote the sequence defined by $v_{n}(x)=u_{\varepsilon_{n}}\left(x_{n}+\varepsilon_{n} x\right)$. Then, there exists a positive function $v \in H^{1}\left(\mathbb{R}^{N}\right) \cap C^{1}\left(\mathbb{R}^{N}\right)$ such that

$$
-\Delta v+V(\bar{x}) v=K(\bar{x}) f(v)
$$

$v$ achieves a maximum at 0 and, along a subsequence,

$$
v_{n} \stackrel{C_{\text {loc }}^{1}\left(\mathbb{R}^{N}\right)}{\longrightarrow} v .
$$


Proof. By Lemma 13, we infer the existence of a cluster point $v \in H^{1}\left(\mathbb{R}^{N}\right)$ of $v_{n}=u_{\varepsilon_{n}}\left(x_{n}+\varepsilon_{n} \cdot\right)$ in $C_{\text {loc }}^{1}\left(\mathbb{R}^{N}\right)$. Since $x_{n} \rightarrow \bar{x}$ in $\Lambda, v$ solves (5.17) and as $v_{n}$ attains a maximum at $0, v$ also achieves a maximum at 0 . We now deduce from Lemma 17 that

$$
f\left(v_{n}(0)\right) / v_{n}(0) \geq V\left(x_{n}\right) / K\left(x_{n}\right)
$$

so that

$$
f(v(0)) / v(0) \geq V(\bar{x}) / K(\bar{x})
$$

and hence $v(0)>0$.

Remark 19. If moreover $f \in C^{k, 1}(\mathbb{R}), V$ and $K$ are of class $C^{k, \alpha}$, for some $k \geq 0$ and $\alpha>0$, similar regularity estimates then yield the convergence $v_{n} \rightarrow v$ in $C_{\mathrm{loc}}^{k+2}\left(\mathbb{R}^{N}\right)$.

Proposition 20. Suppose the assumptions of Lemma 12 are satisfied and assume $u_{\varepsilon} \in H_{\mathrm{loc}}^{1}(\Omega)$ is the positive solution of (4.3) obtained in that lemma. Let $x_{\varepsilon}$ denote a local maximum point of $u_{\varepsilon}$ and assume moreover that all the cluster points of the set $\left\{x_{\varepsilon} \mid 0<\varepsilon \leq \varepsilon_{0}\right\}$ are inside $\Lambda$. Then, there exists $C>0$ such that

$$
u_{\varepsilon}\left(x_{\varepsilon}+\varepsilon y\right) \leq \frac{C}{|y|^{(N-1) / 2}}+o(1),
$$

uniformly in y over compact subsets as $\varepsilon \rightarrow 0$.

Proof. The proof is a direct consequence of Proposition 4 and Proposition 18, taking also the boundedness of the concentration function $\mathcal{C}$, defined by (3.3), in $\Lambda$ into account.

\subsection{Uniform convergence on $\partial \Lambda$}

The main consequence of the previous analysis of sequences of rescaled solutions is the following estimate on the boundary of $\Lambda$. As already discussed, this estimate is crucial in our approach.

Proposition 21. Suppose that $f: \mathbb{R}^{+} \rightarrow \mathbb{R}^{+}$is a continuous function satisfying $\left(f_{1}\right)-\left(f_{4}\right)$ and $V, K: \Omega \rightarrow \mathbb{R}^{+}$are continuous functions. Assume that the open set $\Lambda \subset \subset \Omega$ satisfies (4.11) and $g: \Omega \times \mathbb{R}^{+} \rightarrow \mathbb{R}$ is defined by (4.2). If $N=2$ and $\Omega$ is unbounded, assume moreover that $V$ satisfies

$$
\liminf _{|x| \rightarrow \infty} V(x)|x|^{2}>0 .
$$

Then, the family $\left(u_{\varepsilon}\right)_{\varepsilon} \subset H_{\mathrm{loc}}^{1}(\Omega)$ of positive solutions of (4.3) obtained in Lemma 12 satisfies

$$
\lim _{\varepsilon \rightarrow 0} \sup _{x \in \partial \Lambda} u_{\varepsilon}(x)=0
$$


Proof. Suppose by contradiction that there exist sequences $\left(\varepsilon_{n}\right)_{n} \subset \mathbb{R}^{+}$ and $\left(x_{n}\right)_{n} \subset \partial \Lambda$ such that $\varepsilon_{n} \rightarrow 0$ and

$$
\liminf _{n \rightarrow \infty} u_{\varepsilon_{n}}\left(x_{n}\right)>0 .
$$

Then, going to a subsequence if necessary, $x_{n} \rightarrow \bar{x} \in \partial \Lambda$ and we deduce from Proposition 16 that

$$
\liminf _{n \rightarrow \infty} \varepsilon_{n}^{-N} \mathcal{J}\left(u_{\varepsilon_{n}}\right) \geq \mathcal{C}(\bar{x})
$$

contradicting the energy estimate of Lemma 12.

\section{Solutions of the initial problem}

In this section, we prove that for $\varepsilon$ small enough, the solutions of the modified problem (4.3) do solve the initial problem (2.1). Theorem 1 stated in the introduction is a particular case of the following more general result.

Theorem 22. Suppose $\Omega \subset \mathbb{R}^{N}$ is a regular bounded or exterior domain. Let $V, K \in C\left(\Omega, \mathbb{R}^{+}\right)$satisfy $\left(\mathcal{G}_{f, \partial \Omega}\right)$ if $\partial \Omega \neq \emptyset$ and one set $\left(\mathcal{G}_{f, \infty}^{i}\right)$ of growth conditions if $\Omega$ is unbounded. Let $\Lambda \subset \subset \Omega$ be open and bounded and assume

$$
\inf _{x \in \Lambda} \mathcal{C}(x)<\inf _{x \in \partial \Lambda} \mathcal{C}(x),
$$

where $\mathcal{C}$ is defined by (3.3). Then there exists $\varepsilon_{0}>0$ such that for every $0<\varepsilon<\varepsilon_{0}$, the Dirichlet problem (2.1) has at least one positive solution $u_{\varepsilon}$.

We already know from Lemma 12 that the modified problem (4.3) possesses a positive solution $u_{\varepsilon}$. We will prove that for $\varepsilon$ small enough, this solution actually solves (2.1). Our arguments rely on the construction of suitable comparison functions in order to obtain good decay estimates on the solution $u_{\varepsilon}$ at infinity or close to $\partial \Omega$. These are worked out in the next subsections.

\subsection{Maximum principle}

As in the previous section, $\Omega \subset \mathbb{R}^{N}$ is assumed to have a bounded $C^{1, \alpha}$ boundary. We first define a weak notion of upper and lower solutions for the linear operator $L_{W, \varepsilon}$ defined formally by

$$
L_{W, \varepsilon} u=-\varepsilon^{2} \Delta u+W(x) u,
$$

where $W$ is a continuous nonnegative function and $\varepsilon>0$. 
Definition 23. Let $\Omega \subset \mathbb{R}^{N}$ be a domain and $W \in C(\Omega)$ be nonnegative. A function $v \in W_{\mathrm{loc}}^{1,1}(\Omega)$ is a lower solution of the linear operator $L_{W, \varepsilon}$, formally defined by (6.2) where $\varepsilon>0$, if for every $\varphi \in C_{c}^{\infty}(\Omega)$ such that $\varphi \geq 0$,

$$
\int_{\Omega}\left(\varepsilon^{2} \nabla v \cdot \nabla \varphi+W(x) v \varphi\right) \leq 0 .
$$

A function $v \in H_{\mathrm{loc}}^{1}(\Omega)$ is an upper solution of $L_{W, \varepsilon}$ if $-v$ is a lower solution.

The use of weak solutions is justified by Remark 11. We next state a maximum principle associated to this class of weak solutions.

Proposition 24. Let $\Omega \subset \mathbb{R}^{N}$ be a regular bounded or exterior domain and $L_{W, \varepsilon}$ be the linear operator formally defined by (6.2) where $\varepsilon>0$ and $W \in C(\Omega ; \mathbb{R})$ is nonnegative. Assume that

1. $u \in H_{\mathrm{loc}}^{1}(\Omega)$ is a lower solution of $L_{W, \varepsilon}$;

2. $\nabla u_{+} \in L^{2}(\Omega)$;

3. if $N=2$ and $\Omega$ is unbounded,

$$
\int_{\Omega} \frac{u_{+}^{2}}{1+|x|^{2}}<\infty
$$

Then, if $u_{+}=0$ on $\partial \Omega$, we have $u_{+}=0$ in $\Omega$.

Remark 25. The hypothesis $u \in H_{\mathrm{loc}}^{1}$ together with the summability condition $\nabla u_{+} \in L^{2}(\Omega)$, imply that $u_{+} \in H^{1}(U)$ where $U$ is a bounded neighbourhood of the boundary $\partial \Omega$. Therefore, by the Sobolev trace embedding, $u_{+}$has a trace on $\partial \Omega$.

Remark 26. When $N>2$ or $\Omega$ is bounded, the assumption (3) is indeed unnecessary since in fact, it is a consequence of Hardy's inequality.

Proof of Proposition 24. First notice that since $u \in H_{\text {loc }}^{1}(\Omega)$, the variational inequality (6.3) holds in fact for every $\varphi \in H_{c}^{1}(\Omega)$, that is the set of compactly supported functions of $H^{1}(\Omega)$. Let $\eta_{\lambda}$ be the cut-off function defined by $(4.7)$ in the proof of Lemma 6 . Taking $\left(1-\eta_{\lambda}\right) u_{+}$as test function in $(6.3)$, we get

$$
\int_{\Omega}\left(1-\eta_{\lambda}\right)\left(\varepsilon^{2}\left|\nabla u_{+}\right|^{2}+W(x) u_{+}^{2}\right) \leq \int_{\Omega} \nabla u_{+} \cdot \nabla \eta_{\lambda} u_{+}
$$


Since $u_{+}=0$ on $\partial \Omega$, taking the assumptions (2) and (3) into account, we may argue as in the proof of Lemma 6 to estimate the right-hand side of this last inequality. We then infer this right-hand side is of order $1 / \lambda$ as $\lambda \rightarrow \infty$. On the other hand, it is clear that $\eta_{\lambda} \rightarrow 0$ as $\lambda \rightarrow \infty$. Therefore, as $W$ is nonnegative, Fatou's Lemma yields

$$
\int_{\Omega} \varepsilon^{2}\left|\nabla u_{+}\right|^{2}+W(x) u_{+}^{2} \leq \liminf _{\lambda \rightarrow \infty} \int_{\Omega} \nabla u_{+} \cdot \nabla \eta_{\lambda} u_{+}=0 .
$$

Hence, we conclude that $u_{+}=0$.

\subsection{Comparison functions}

We first consider comparison functions close to the boundary. Along a smooth boundary, it is possible to construct a harmonic function that decay uniformly when approaching the boundary.

Proposition 27. Let $\Omega$ be a regular bounded or exterior domain in $\mathbb{R}^{N}$ with $N \geq 2$. Assume $\Lambda \subset \Omega$ is a regular subdomain such that $\bar{\Lambda} \subset \Omega$. Then, there exists a function $\psi_{b}$ such that $\nabla \psi_{b} \in L^{2}(\Omega)$,

$$
-\Delta \psi_{b} \geq 0 \quad \text { and } \quad \psi_{b}(x) \leq C \frac{d(x, \partial \Omega)}{1+d(x, \partial \Omega)}
$$

on $\Omega \backslash \bar{\Lambda}$,

$$
\psi_{b}=1
$$

on $\Lambda$ and $\psi_{b}(x)=0$ for $x \in \partial \Omega$.

Proof. Choose $U \subset \Omega$ such that $U \cap \Lambda=\emptyset, U$ is bounded with a regular boundary and $\partial \Omega \subset \partial U$. Define $\psi_{b}: \bar{\Omega} \backslash \Lambda \rightarrow \mathbb{R}$ by

$$
\begin{cases}\Delta \psi_{b}=0 & \text { in } U \\ \psi_{b}=0 & \text { on } \partial \Omega \\ \psi_{b}=1 & \text { on } \partial U \backslash \partial \Omega . \\ \psi_{b}=1 & \text { on } \Omega \backslash U .\end{cases}
$$

The function $\psi_{b}$ is clearly subharmonic. The regularity hypothesis on $\Omega$ and $U$ imply that $\psi_{b} \in C^{1, \alpha}(\bar{U})$. Therefore, we have

$$
\psi_{b}(x) \leq C \frac{d(x, \partial \Omega)}{1+d(x, \partial \Omega)}
$$

for $x \in \Omega \backslash \Lambda$ and $\nabla \psi_{b} \in L^{2}(\Omega)$. 
The next proposition deals with comparison functions at infinity.

Proposition 28. Let $N \geq 1, U \subset \mathbb{R}^{N}$ be unbounded and $W \in C\left(U ; \mathbb{R}^{+}\right)$. Assume either

(i) $\liminf _{x \rightarrow \partial U} W(x)>0$ and there exists $\alpha<2$ such that

$$
\begin{gathered}
\liminf _{|x| \rightarrow \infty} W(x)|x|^{\alpha}>0 \\
\text { and } \psi_{\infty}(x)=\exp \left(-\lambda\left(1+|x|^{2}\right)^{1 / 2-\alpha / 4}\right) \text {, where } \lambda>0 \text {, or }
\end{gathered}
$$

(ii) $\liminf _{x \rightarrow \partial U} W(x)>0$,

$$
\liminf _{|x| \rightarrow \infty} W(x)|x|^{2}>0,
$$

and $\psi_{\infty}(x)=\left(1+|x|^{2}\right)^{-\lambda / 2}$, where $\lambda>0$, or

(iii) $W$ is nonnegative, $N>2$ and $\psi_{\infty}(x)=\left(1+|x|^{2}\right)^{1-N / 2}$.

Then there exists $\varepsilon_{0}>0$ such that if $0<\varepsilon<\varepsilon_{0}$,

$$
-\varepsilon^{2} \Delta \psi_{\infty}+W(x) \psi_{\infty} \geq 0
$$

in $U$.

Proof. Consider the case (i). By assumption, there is $c>0$ such that for $x \in U, W(x) \geq c /(1+|x|)^{\alpha}$. An explicit computation of $-\varepsilon^{2} \Delta \psi_{\infty}$ combined with the previous inequality gives

$$
\begin{aligned}
& -\varepsilon^{2} \Delta \psi_{\infty}+W(x) \psi_{\infty} \\
& \geq\left(\varepsilon^{2} \lambda\left(1-\frac{\alpha}{2}\right)\left(-\lambda\left(1-\frac{\alpha}{2}\right) \frac{|x|^{2}}{1+|x|^{2}}+\frac{N+\left(N-1-\frac{\alpha}{2}\right)|x|^{2}}{2\left(1+|x|^{2}\right)^{3 / 2-\alpha / 4}}\right)+c\right) \frac{\psi_{\infty}}{\left(1+|x|^{2}\right)^{\alpha / 2}} .
\end{aligned}
$$

Since $\alpha<2$, this last expression is positive for every $x \in U$ when $\varepsilon$ is sufficiently small.

Under the assumptions (ii) and (iii), one computes

$$
-\Delta\left(\frac{1}{\left(1+|x|^{2}\right)^{\lambda / 2}}\right)=\frac{\lambda}{\left(1+|x|^{2}\right)^{\lambda / 2+1}}\left(\frac{N-(\lambda-N+2)|x|^{2}}{1+|x|^{2}}\right) .
$$

In case (ii), one concludes as in case (i), while in case (iii), one has even $-\Delta \psi_{\infty} \geq 0$, so that in this case, the conclusion holds for any nonnegative $W$. 


\subsection{Proof of Theorem 22}

To fix the ideas, we work out the proof for an exterior domain $\Omega$ such that $\partial \Omega \neq \emptyset$ and $\left(\mathcal{G}_{f, \infty}^{1}\right)$ holds.

By $\left(\mathcal{G}_{f, \infty}^{1}\right)$ and $\left(\mathcal{G}_{f, \partial \Omega}\right)$, there exist $\lambda>0, \alpha \in[0,2[, \mu>0$ and $\kappa \in(0,1)$ such that

$$
\limsup _{|x| \rightarrow \infty} \frac{f\left(\exp \left(-\lambda|x|^{1-\alpha / 2}\right)\right)}{\exp \left(-\lambda|x|^{1-\alpha / 2}\right)} \frac{K(x)}{V(x)}<\kappa,
$$

and

$$
\limsup _{d(x, \partial \Omega) \rightarrow 0} \frac{f(\mu d(x, \partial \Omega))}{\mu d(x, \partial \Omega)} \frac{K(x)}{V(x)}<\kappa .
$$

Define $\tilde{f}$ by (4.1) according to this choice of $\kappa$. We know from Lemma 12 that the modified problem (4.3) has a positive solution $u_{\varepsilon}$. If for every $x \in \Omega \backslash \Lambda$, we have

$$
\frac{f\left(u_{\varepsilon}(x)\right)}{u_{\varepsilon}(x)} \frac{K(x)}{V(x)} \leq \kappa
$$

then $u_{\varepsilon}$ is a positive solution of the original Dirichlet problem (2.1).

From the assumptions $\left(f_{1}\right),\left(f_{4}\right),\left(\mathcal{G}_{f, \partial \Omega}\right)$ and $\left(\mathcal{G}_{f, \infty}^{1}\right)$, we deduce the existence of $\gamma>0$ sufficiently small, such that, choosing

$$
w(x)=\gamma \exp \left(-\lambda|x|^{1-\alpha / 2}\right) \frac{d(x, \partial \Omega)}{1+d(x, \partial \Omega)},
$$

one has for all $x \in \Omega$,

$$
\frac{f(w(x))}{w(x)} \frac{K(x)}{V(x)} \leq \kappa
$$

Let us write $m_{\varepsilon}=\sup _{x \in \partial \Lambda} u_{\varepsilon}(x)$ and define the auxiliary function $w_{b}$ by

$$
w_{b}(x):=\frac{u_{\varepsilon}(x)}{m_{\varepsilon}}-\psi_{b}(x),
$$

where $\psi_{b}$ is defined in Proposition 27. We infer from the fact that $u_{\varepsilon}$ solves (4.3) and the definition of $g(x, u)$ that

$$
-\Delta w_{b} \leq 0 \quad \text { in } \Omega \backslash \Lambda
$$

Applying the maximum principle for subharmonics, i.e. Proposition 24 with $W \equiv 0$, to $w_{b}$ in $\Omega \backslash \Lambda$, we deduce from Proposition 27 the estimate

$$
u_{\varepsilon}(x) \leq C m_{\varepsilon} \frac{d(x, \partial \Omega)}{1+d(x, \partial \Omega)}
$$

which is valid for every $x \in \Omega \backslash \Lambda$. 
Now, choose $U \subset \Omega$ such that $\Omega \backslash U$ is bounded, $\Lambda \cap U=\emptyset, \bar{U} \subset \Omega$ and $\partial U$ is compact and smooth. It follows from (6.5) that $u_{\varepsilon}$ is bounded on $\partial U$ uniformly in $\varepsilon$.

To get a decay estimate at infinity, we define the auxiliary function $w_{\infty}$ by

$$
w_{\infty}(x):=\frac{u_{\varepsilon}(x)}{m_{\varepsilon}}-C \psi_{\infty}(x)
$$

where $\psi_{\infty}$ is defined in Proposition 28 with $\lambda>0$ as in assumption $\left(\mathcal{G}_{f, \infty}^{1}\right)$ and $C>0$. This time, we observe that for $\varepsilon$ small enough, $w_{\infty}$ is a lower solution of $L_{W, \varepsilon}$ in $U$, where $W(x):=(1-\kappa) V(x)$. Choosing $C$ large enough to ensure that $w_{\infty} \leq 0$ on $\partial U$ and applying again Proposition 24, we get

$$
u_{\varepsilon}(x) \leq C m_{\varepsilon} \psi_{\infty}(x)
$$

for $x \in U$.

As $m_{\varepsilon} \rightarrow 0$ by Proposition 21 and $\Omega \backslash U$ is bounded, combining the estimates (6.5) and (6.6), we finally deduce that for $\varepsilon$ small enough,

$$
u_{\varepsilon}(x) \leq w(x)
$$

for every $x \in \Omega \backslash \Lambda$.

At last, we conclude from $\left(f_{4}\right)$ and (6.4) that for each $x \in \Omega \backslash \Lambda$,

$$
\frac{f\left(u_{\varepsilon}(x)\right)}{u_{\varepsilon}(x)} \frac{K(x)}{V(x)} \leq \frac{f(w(x))}{w(x)} \frac{K(x)}{V(x)} \leq \kappa .
$$

This completes the proof of this case. The arguments being similar when dealing with the assumptions $\left(\mathcal{G}_{f, \infty}^{2}\right)$ or $\left(\mathcal{G}_{f, \infty}^{3}\right)$, we do not repeat them.

Remark 29. The inequality (6.6) provides a decay estimate at infinity for the solution $u_{\varepsilon}$. In particular, this estimate does hold even if $\alpha<0$ in assumption $\left(\mathcal{G}_{f, \infty}^{1}\right)$. If $\alpha=0$, i.e. if $V$ is bounded from below at infinity, we infer the solution decay exponentially fast at infinity. If $\alpha<0$, this decay rate at infinity can be improved. For example, if $V$ grows quadratically fast at infinity, then the solution decay like a Gaussian as $|x| \rightarrow \infty$.

\section{Concentration}

We next investigate the behaviour of the solutions $u_{\varepsilon}$ obtained in Theorem 22 when $\varepsilon \rightarrow 0$. Namely, we prove in this section that the solutions display the features stated in the following theorem. 
Theorem 30. Suppose that the assumptions of Theorem 22 hold. Let $u_{\varepsilon}$ be the positive solution of (1.11) obtained in that theorem and $x_{\varepsilon} \in \Omega$ be such that

$$
u_{\varepsilon}\left(x_{\varepsilon}\right)=\sup _{x \in \Omega} u_{\varepsilon}(x) .
$$

Then, we have

$$
\lim _{\varepsilon \rightarrow 0} \mathcal{C}\left(x_{\varepsilon}\right)=\inf _{x \in \Lambda} \mathcal{C}(x)
$$

and for every $r>0$, there exist $C>0$ and $\varepsilon_{0}>0$ such that for $0<\varepsilon<\varepsilon_{0}$, $u_{\varepsilon}$ has no local maximum outside the ball $B\left(x_{\varepsilon}, \varepsilon r\right)$ and

$$
u_{\varepsilon}(x) \leq C \frac{d(x, \partial \Omega)}{1+d(x, \partial \Omega)} \frac{\varepsilon^{N-2}}{\left(\varepsilon^{2}+\left|x-x_{\varepsilon}\right|^{2}\right)^{\frac{N-2}{2}}} .
$$

If we assume in addition that

$$
\liminf _{d(x, \partial \Omega) \rightarrow 0} V(x)>0 \quad \text { and } \quad \liminf _{|x| \rightarrow \infty} V(x)|x|^{2}>0,
$$

then for every $\lambda>0$, there exist $C>0$ and $\varepsilon_{0}>0$ such that for $0<\varepsilon<\varepsilon_{0}$, one has

$$
u_{\varepsilon}(x) \leq C \frac{d(x, \partial \Omega)}{1+d(x, \partial \Omega)} \frac{\varepsilon^{\lambda}}{\left(\varepsilon^{2}+\left|x-x_{\varepsilon}\right|^{2}\right)^{\lambda / 2}},
$$

while if

$$
\liminf _{d(x, \partial \Omega) \rightarrow 0} V(x)>0 \text { and } \liminf _{|x| \rightarrow \infty} V(x)|x|^{\alpha}>0
$$

for some $\alpha \in] 0,2\left[\right.$, then for every $\lambda>0$, there exist $C>0$ and $\varepsilon_{0}>0$ such that for $0<\varepsilon<\varepsilon_{0}$,

$$
u_{\varepsilon}(x) \leq C \frac{d(x, \partial \Omega)}{1+d(x, \partial \Omega)} \exp \left(-\lambda\left|\frac{x-x_{\varepsilon}}{\varepsilon}\right|^{1-\alpha / 2}\right) .
$$

When $\Omega=\mathbb{R}^{N}$ the preceding holds provided $d(x, \partial \Omega) /(1+d(x, \partial \Omega))$ is replaced by 1 .

Theorem 2 stated in the introduction concerning the particular case $f(u)=u^{p}$ clearly follows from this more general result. The proof of Theorem 30 is divided in two steps. We first investigate the behaviour of the maxima of $u_{\varepsilon}$. Then, the second and main step is the construction of barrier functions, see below for a precise definition, which basically consist in families of comparison functions that provide uniform decay properties as $\varepsilon \rightarrow 0$.

Observe that in contrast with del Pino-Felmer Theorem stated in the introduction, we cannot ensure the uniqueness of the maximum of $u_{\varepsilon}$. This is due to the lack of regularity of $f, V$ and $K$. When stronger regularity assumptions are made on those functions, one recovers solutions with a single maximum as in the above cited theorem, see Remark 35 below. 


\subsection{Local and global maxima}

A first thing noteworthy in the study of maxima of $u_{\varepsilon}$ is that the global maximum is always attained in $\bar{\Lambda}$.

Proposition 31. Suppose that $f: \mathbb{R}^{+} \rightarrow \mathbb{R}^{+}, V, K: \Omega \rightarrow \mathbb{R}^{+}$are continuous functions, $\Lambda \subset \subset \Omega$ and $g: \Omega \times \mathbb{R}^{+} \rightarrow \mathbb{R}$ is defined by (4.2). Let $u_{\varepsilon} \in H_{\mathrm{loc}}^{1}(\Omega)$ be a nonnegative solution of $(4.3)$ such $\nabla u_{\varepsilon} \in L^{2}(\Omega)$. Then there exists $x_{\varepsilon} \in \bar{\Lambda}$ such that

$$
u_{\varepsilon}\left(x_{\varepsilon}\right)=\sup _{x \in \Omega} u_{\varepsilon}(x) .
$$

Proof. Since the solution $u_{\varepsilon} \in C^{1, \alpha}(\Omega)$ by Proposition 10 and $\bar{\Lambda} \subset \Omega$ is compact, there exists $x_{\varepsilon} \in \bar{\Lambda}$ such that

$$
u_{\varepsilon}\left(x_{\varepsilon}\right)=\max _{x \in \bar{\Lambda}} u_{\varepsilon}(x) .
$$

Now observe that by definition of $g$ in (4.1), one has,

$$
-\varepsilon^{2} \Delta u_{\varepsilon}+(1-\kappa) V(x) u_{\varepsilon} \leq 0
$$

in $\Omega \backslash \Lambda$, where $\kappa<1$. Hence, using the maximum principle which applies because $\nabla u_{\varepsilon} \in L^{2}(\Omega)$, we infer that for every $x \in \Omega \backslash \bar{\Lambda}$,

$$
u_{\varepsilon}(x) \leq \sup _{x \in \partial \Omega \cup \partial \Lambda} u_{\varepsilon}(x) \leq \sup _{x \in \bar{\Lambda}} u_{\varepsilon}(x)=u_{\varepsilon}\left(x_{\varepsilon}\right) .
$$

In the sequel of this paragraph, we investigate the localization of the maxima of $u_{\varepsilon}$ in $\Omega$. Our first observation is that the maximum points of the solution obtained in Theorem 22 are all located in $\Lambda$.

Proposition 32. Suppose the assumptions of Theorem 22 hold. Let $u_{\varepsilon}$ be the positive solution of (1.11) obtained in that theorem and $x_{\varepsilon} \in \Omega$ be a local maximum point of $u_{\varepsilon}$. Then $x_{\varepsilon} \in \Lambda$.

Proof. The proof follows from the penalization procedure and Lemma 17. Indeed, if $x_{\varepsilon} \in \Omega$ is a local maximum point of $u_{\varepsilon}$, then, as $u_{\varepsilon}$ is strictly positive, Lemma 17 implies

$$
\frac{f\left(u_{\varepsilon}\left(x_{\varepsilon}\right)\right)}{u_{\varepsilon}\left(x_{\varepsilon}\right)} \frac{K\left(x_{\varepsilon}\right)}{V\left(x_{\varepsilon}\right)} \geq 1
$$

But on the other hand, since $u_{\varepsilon}$ solves both (4.3) and (1.11), we have for every $x \in \Omega \backslash \Lambda$,

$$
\frac{f\left(u_{\varepsilon}(x)\right)}{u_{\varepsilon}(x)} \frac{K(x)}{V(x)} \leq \kappa
$$

with $\kappa<1$. 
Our second fact is that any converging sequence of maximum points of $u_{\varepsilon}$ does converge to a minimum point of $\mathcal{C}$ in $\Lambda$. This obviously implies that as $\varepsilon \rightarrow 0$, the maxima of $u_{\varepsilon}$ occur close to minima of $\mathcal{C}$.

Proposition 33. Suppose that $\Lambda \subset \subset \Omega$ and the assumptions of Lemma 12 are satisfied. Let $\left(\varepsilon_{n}\right)_{n} \subset \mathbb{R}^{+}$be such that $\varepsilon_{n} \rightarrow 0$ as $n \rightarrow \infty$ and $\left(u_{\varepsilon_{n}}\right)_{n} \subset$ $H_{\text {loc }}^{1}(\Omega)$ be the corresponding sequence of positive solutions of (4.3) obtained in Lemma 12. If $\left(y_{n}\right)_{n} \subset \Lambda$ is a sequence of local maximum points of $u_{\varepsilon_{n}}$, then

$$
\lim _{n \rightarrow \infty} \mathcal{C}\left(y_{n}\right)=\inf _{x \in \Lambda} \mathcal{C}(x) .
$$

Proof. Assume by contradiction that the conclusion is false. Hence, by compactness, we infer that, up to a subsequence, $\left(y_{n}\right)_{n}$ converges to $\bar{y} \in \bar{\Lambda}$ such that

$$
\mathcal{C}(\bar{y})>\inf _{x \in \Lambda} \mathcal{C}(x)
$$

On the one hand, we deduce from Proposition 16 and Lemma 17 that

$$
\liminf _{n \rightarrow \infty} \varepsilon_{n}^{-N} \mathcal{J}_{\varepsilon_{n}}\left(u_{\varepsilon_{n}}\right) \geq \mathcal{C}(\bar{y})
$$

On the other hand, by Lemma $12, u_{\varepsilon_{n}}$ does satisfy

$$
\limsup _{n \rightarrow \infty} \varepsilon_{n}^{-N} \mathcal{J}_{\varepsilon_{n}}\left(u_{\varepsilon_{n}}\right) \leq \inf _{x \in \Lambda} \mathcal{C}(x) .
$$

This contradicts (7.4) and concludes the proof.

We next prove that local maxima are essentially unique in the sense that they get closer and closer to the global one as $\varepsilon \rightarrow 0$. Therefore, even if $u_{\varepsilon}$ can have more than one local maximum, the solution is a perturbation of a solution with a single local (hence global) maximum.

Proposition 34. Suppose the assumptions of Theorem 22 hold. Let $u_{\varepsilon}$ be the positive solution of (1.11) obtained in that theorem and $x_{\varepsilon} \in \Omega$ be such that

$$
u_{\varepsilon}\left(x_{\varepsilon}\right)=\sup _{x \in \Omega} u_{\varepsilon}(x) .
$$

Then, for every $r>0$, there exists $\varepsilon_{0}>0$ such that for every $0<\varepsilon<\varepsilon_{0}, u_{\varepsilon}$ has no local maxima in $\Lambda \backslash B\left(x_{\varepsilon}, \varepsilon r\right)$.

Proof. Let $\left(x_{\varepsilon}\right)_{\varepsilon} \subset \Omega$ be global maximum points of $u_{\varepsilon}$. We argue by contradiction, assuming the existence of sequences $\left(y_{n}\right)_{n} \subset \Omega$ and $\left(\varepsilon_{n}\right)_{n} \subset \mathbb{R}^{+}$ such that $u_{\varepsilon_{n}}$ attains a local maximum at $y_{n}, \varepsilon_{n} \rightarrow 0$ as $n \rightarrow \infty$ and

$$
\liminf _{n \rightarrow \infty} \frac{\left|x_{\varepsilon_{n}}-y_{n}\right|}{\varepsilon_{n}}>0
$$


By Proposition 32, we may assume without loss of generality that $\left(x_{\varepsilon_{n}}\right)_{n} \subset \Lambda$, $\left(y_{\varepsilon_{n}}\right)_{n} \subset \Lambda$,

$$
\liminf _{n \rightarrow \infty} u_{\varepsilon_{n}}\left(x_{\varepsilon_{n}}\right)>0 \text { and } \quad \liminf _{n \rightarrow \infty} u_{\varepsilon_{n}}\left(y_{\varepsilon_{n}}\right)>0
$$

Since $\bar{\Lambda}$ is compact, going to a subsequence if necessary, we may also assume that $x_{\varepsilon_{n}} \rightarrow \bar{x}$ and $y_{n} \rightarrow \bar{y}$. Now, if

$$
\limsup _{n \rightarrow \infty} \frac{\left|x_{\varepsilon_{n}}-y_{\varepsilon_{n}}\right|}{\varepsilon_{n}}=\infty
$$

Proposition 16 applies. We therefore conclude that

$$
\liminf _{n \rightarrow \infty} \varepsilon_{n}^{-N} \mathcal{J}_{\varepsilon_{n}}\left(u_{\varepsilon_{n}}\right) \geq \mathcal{C}(\bar{x})+\mathcal{C}(\bar{y}) \geq 2 \inf _{x \in \Lambda} \mathcal{C}(x),
$$

while by Lemma 12, we know that

$$
\limsup _{n \rightarrow \infty} \varepsilon_{n}^{-N} \mathcal{J}_{\varepsilon_{n}}\left(u_{\varepsilon_{n}}\right) \leq \inf _{x \in \Lambda} \mathcal{C}(x) .
$$

Since $\inf _{\Lambda} \mathcal{C}>0$, this brings a contradiction. Therefore,

$$
\limsup _{n \rightarrow \infty} \frac{\left|x_{\varepsilon_{n}}-y_{n}\right|}{\varepsilon_{n}}<\infty
$$

Consider now the sequences $\left(v_{n}\right)_{n}$ and $\left(z_{n}\right)_{n}$ defined by $v_{n}(z)=u_{\varepsilon_{n}}\left(x_{\varepsilon_{n}}-\varepsilon_{n} z\right)$ and $z_{n}=\left(x_{\varepsilon_{n}}-y_{n}\right) / \varepsilon_{n}$. Since $z_{n}$ is a local maximum of $v_{n}, \nabla v_{n}\left(z_{n}\right)=0$. By Proposition 18 and (7.6), up to subsequences, we have $\nabla v_{n} \rightarrow \nabla v$ uniformly on compact subsets, where $v$ is a solution of $(5.17)$, and $z_{n} \rightarrow z \in \mathbb{R}^{N}$. Therefore $\nabla v(z)=\lim _{n \rightarrow \infty} \nabla v_{n}\left(z_{n}\right)=0$, so that by Lemma $3, z=0$, i.e.

$$
\lim _{n \rightarrow \infty} \frac{\left|x_{\varepsilon_{n}}-y_{n}\right|}{\varepsilon_{n}}=0
$$

in contradiction with (7.5).

Remark 35. We may state a stronger conclusion in Proposition 34 when $f$ is locally Lipschitz continuous and $V$ and $K$ are both Hölder continuous inside $\Lambda$. Indeed, in this case, the sequence $\left(v_{n}\right)_{n}$ of rescaled solutions defined by $v_{n}(x)=u_{\varepsilon_{n}}\left(x_{\varepsilon_{n}}+\varepsilon_{n} x\right)$ converges for the $C_{\mathrm{loc}}^{2}$ topology, see Remark 19, and the limit function $v$ has a nondegenerate maximum at 0 . Hence there exists $\varepsilon_{0}>0$ such that $x_{\varepsilon}$ is the unique local maximum of $u_{\varepsilon}$ in $\Omega$. 


\subsection{Elliptic inequation outside small balls}

All the previous results allow to prove the following inequality which will be useful to get concentration estimates.

Proposition 36. Suppose the assumptions of Theorem 22 hold. Let $u_{\varepsilon}$ be the positive solution of (1.11) obtained in that theorem and $x_{\varepsilon} \in \Omega$ be such that

$$
u_{\varepsilon}\left(x_{\varepsilon}\right)=\sup _{x \in \Omega} u_{\varepsilon}(x)
$$

Then there exists $r_{0}>0$ such that for every $r>r_{0}$, there exists $\varepsilon_{r}>0$ such that for every $\varepsilon \in] 0, \varepsilon_{r}[$,

$$
-\varepsilon^{2} \Delta u_{\varepsilon}+(1-\kappa) V(x) u_{\varepsilon} \leq 0
$$

in $\Omega \backslash B\left(x_{\varepsilon}, \varepsilon r\right)$, where $\kappa<1$ is defined in (4.1).

Proof. First, notice that by the compactness of $\bar{\Lambda} \subset \Omega$, the continuity of $V$ and $K$ and the assumption $\left(f_{1}\right)$, we infer there exists $a>0$ such that

$$
\frac{f(a)}{a} \leq \kappa \frac{V(x)}{K(x)}
$$

for every $x \in \Lambda$. On the other hand, by Proposition 20 and Proposition 34, we may take $r>0$ large enough such that for $\varepsilon \rightarrow 0$,

$$
u_{\varepsilon}\left(x_{\varepsilon}+\varepsilon y\right) \leq a
$$

for $|y|=r$ and $u_{\varepsilon}$ has no local maximum in $\Lambda \backslash B\left(x_{\varepsilon}, \varepsilon r\right)$. Moreover, we know from Proposition 21 that

$$
\max _{x \in \partial \Lambda} u_{\varepsilon}(x) \rightarrow 0 \quad \text { as } \varepsilon \rightarrow 0 .
$$

Hence, one can assume that for small $\varepsilon, u_{\varepsilon}(x) \leq a$ for every $x \in \Lambda \backslash B\left(x_{\varepsilon}, \varepsilon r\right)$. Taking (7.7) into account, we now infer from $\left(f_{4}\right)$ that

$$
g\left(x, u_{\varepsilon}(x)\right)=K(x) f\left(u_{\varepsilon}(x)\right) \leq \kappa V(x) u_{\varepsilon}(x)
$$

for $x \in \Lambda \backslash B\left(x_{\varepsilon}, \varepsilon r\right)$. On the other hand, by the definition of the penalization, we have

$$
g\left(x, u_{\varepsilon}(x)\right) \leq \kappa V(x) u_{\varepsilon}(x)
$$

for $x \in \Omega \backslash \Lambda$.

Therefore $u_{\varepsilon}$ satisfies the desired inequality for $\varepsilon$ small enough. 


\subsection{Barrier functions}

Proposition 36 implies that for $r$ large and $\varepsilon$ small enough, $\left(u_{\varepsilon}\right)_{\varepsilon}$ is a family of lower solutions of $-\varepsilon^{2} \Delta+W$ in $\Omega \backslash B\left(x_{\varepsilon}, r \varepsilon\right)$, where $W=(1-\kappa) V$ and $x_{\varepsilon}$ is a global maximum point of $u_{\varepsilon}$. Then, arguing as in the proof of Theorem 22, one can easily obtain estimates for $u_{\varepsilon}$ in $\Omega \backslash B\left(x_{\varepsilon}, r \varepsilon\right)$ if we can compare $u_{\varepsilon}$ with a convenient upper solution in this set. This motivates the following definition.

Definition 37. Let $\Omega \subset \mathbb{R}^{N}$ be a regular bounded or exterior domain and $L_{W, \varepsilon}$ be the linear operator formally defined by (6.2) where $\varepsilon>0$ and $W \in$ $C(\Omega ; \mathbb{R})$ is nonnegative. We say that the set $\left(w_{\varepsilon}\right)_{\varepsilon} \subset H_{\mathrm{loc}}^{1}\left(\Omega \backslash B\left(x_{\varepsilon}, r \varepsilon\right)\right)$, where $r>0$ and $\left(x_{\varepsilon}\right)_{\varepsilon} \subset \Omega$, is a family of barrier functions for $W$ if there exists $\varepsilon_{0}>0$ such that for every $0<\varepsilon<\varepsilon_{0}$,

1. $B\left(x_{\varepsilon}, r \varepsilon\right) \subset \Omega$;

2. $w_{\varepsilon}$ is an upper solution of $L_{W, \varepsilon}$ in $\Omega \backslash B\left(x_{\varepsilon}, r \varepsilon\right)$;

3. $\nabla w_{\varepsilon} \in L^{2}\left(\Omega \backslash B\left(x_{\varepsilon}, r \varepsilon\right)\right)$;

4. $w_{\varepsilon} \geq 1$ on $\partial B\left(x_{\varepsilon}, r \varepsilon\right)$.

As a basic example, the constant functions $w_{\varepsilon} \equiv 1$ form a family of barrier functions for any nonnegative potential $W$.

Remark 38. One easily checks that if $\left(w_{\varepsilon}\right)_{\varepsilon}$ is a family of barrier functions for $W \in C(\Omega ; \mathbb{R})$ and if $\bar{W} \in C(\Omega ; \mathbb{R})$ satisfies $\bar{W} \geq W$, then $\left(w_{\varepsilon}\right)_{\varepsilon}$ is a family of barrier functions for $\bar{W}$. Note also that if $\lambda \geq 0$, then $\left(w_{\lambda \varepsilon}\right)_{\varepsilon}$ is a family of barrier functions for $\lambda^{-2} W$.

As mentioned above, the main interest of a family of barrier functions is to deduce estimates for the solutions $\left(u_{\varepsilon}\right)_{\varepsilon}$ obtained in Theorem 22 . These estimates will be obtained through the following proposition.

Proposition 39. Let $\Omega \subset \mathbb{R}^{N}$ be a regular bounded or exterior domain and $L_{W, \varepsilon}$ be the linear operator formally defined by (6.2) where $\varepsilon>0$ and $W \in C(\Omega ; \mathbb{R})$ is nonnegative. Assume $w_{\varepsilon} \in H_{\text {loc }}^{1}\left(\Omega \backslash B\left(x_{\varepsilon}, r \varepsilon\right)\right)$, where $r>0$ and $\left(x_{\varepsilon}\right)_{\varepsilon} \subset \Omega$, is a familly of positive barrier functions. If $v_{\varepsilon} \in \mathcal{H}_{\varepsilon}$ is a lower solution of $L_{W, \varepsilon}$ in $\Omega \backslash B\left(x_{\varepsilon}, r \varepsilon\right)$ such that

$$
\int_{\Omega} \frac{\left|v_{\varepsilon}\right|^{2}}{1+|x|^{2}}<\infty
$$

if $N=2$ and $\Omega$ is unbounded, and

$$
v_{\varepsilon} \leq c_{\varepsilon}
$$

on $\partial B\left(x_{\varepsilon}, r \varepsilon\right)$, then for every $\left.\varepsilon \in\right] 0, \varepsilon_{0}[$, we have

$$
v_{\varepsilon} \leq c_{\varepsilon} w_{\varepsilon} \quad \text { in } \Omega \backslash B\left(x_{\varepsilon}, r \varepsilon\right) \text {. }
$$


Proof. The proof follows immediately by applying Proposition 24 to the functions $v_{\varepsilon} / c_{\varepsilon}-w_{\varepsilon}$ taking Definition 37 into account.

The main concern in this section is to obtain uniform estimates as $\varepsilon \rightarrow 0$ so that we have to select carefully the family of barrier functions in our application of Proposition 39.

Assuming that the potential $V$ is positive, we directly deduce a rough asymptotic behaviour using constant barrier functions. However, Remark 38 suggests that the construction of barriers depends on the asymptotic behaviour of $V$ so that we may hope an improvement of these basic estimates by choosing a suitable family of barrier functions. In fact, without further restrictions on the potential, the constant barriers can always be replaced by harmonic barriers.

Proposition 40. Let $N \geq 2$ and $\Omega \subset \mathbb{R}^{N}$ be a domain. Assume $\left(x_{\varepsilon}\right)_{\varepsilon} \subset \Omega$ is relatively compact in $\Omega$. Then, the family $\left(H_{\varepsilon}\right)_{\varepsilon} \subset H_{\mathrm{loc}}^{1}\left(\Omega \backslash B\left(x_{\varepsilon}, r \varepsilon\right)\right)$ defined by

$$
H_{\varepsilon}(x)=\frac{\left(\varepsilon \sqrt{1+r^{2}}\right)^{N-2}}{\left(\varepsilon^{2}+\left|x-x_{\varepsilon}\right|^{2}\right)^{N / 2-1}}
$$

is a family of barrier functions for any nonnegative $W \in C(\Omega ; \mathbb{R})$.

Proof. Notice that $-\Delta H_{\varepsilon} \geq 0$ on $\Omega \backslash\left\{x_{\varepsilon}\right\}$. All the properties follow then from straightforward computations.

When $N=2$, we recover the constant barriers $w_{\varepsilon} \equiv 1$ while for $N>2$, the barrier functions provide a polynomial decay to 0 at infinity. This control at infinity can be improved by either exponential or polynomial (of any order) barriers provided we assume further that

$$
\liminf _{|x| \rightarrow \infty} W(x)|x|^{\alpha}>0 \quad \text { for some } \alpha \in[0,2] .
$$

If $W$ satisfies (7.8) for some $\alpha \in 0,2$ [, then there exists families of barrier functions for $W$ that decay exponentially fast at infinity.

Proposition 41. Let $N \geq 2, \Omega \subset \mathbb{R}^{N}$ be an unbounded domain, $r>0$ and $\left(x_{\varepsilon}\right)_{\varepsilon} \subset \Omega$ be relatively compact in $\Omega$. Assume that $W \in C(\Omega ; \mathbb{R})$ is a positive potential satisfying (7.8) for some $\alpha \in] 0,2[$ and

$$
\liminf _{d(x, \partial \Omega) \rightarrow 0} W(x)>0,
$$

if $\partial \Omega \neq \emptyset$. Then, for any $\lambda>0$, there exists $r_{0}>0$ such that $\left(E_{\alpha, \lambda, \varepsilon}\right)_{\varepsilon} \subset$ $H_{\text {loc }}^{1}\left(\Omega \backslash B\left(x_{\varepsilon}, r \varepsilon\right)\right)$ defined by

$$
E_{\alpha, \lambda, \varepsilon}(x)=\exp \left(\lambda r^{1-\alpha / 2}-\lambda\left|\frac{x-x_{\varepsilon}}{\varepsilon}\right|^{1-\alpha / 2}\right)
$$

is a family of barrier functions for $W$ when $r>r_{0}$. 
Proof. Let us write for simplicity $E_{\varepsilon}(x)=E_{\alpha, \lambda, \varepsilon}(x)$. We then compute for $x \in \Omega \backslash B\left(x_{\varepsilon}, r \varepsilon\right)$,

$$
-\varepsilon^{2} \Delta E_{\varepsilon}(x)+W(x) E_{\varepsilon}(x) \geq\left(-\lambda^{2}\left(1-\frac{\alpha}{2}\right)^{2} \frac{\varepsilon^{\alpha}}{\left|x-x_{\varepsilon}\right|^{\alpha}}+W(x)\right) E(x) .
$$

By assumption, since $\left(x_{\varepsilon}\right)_{\varepsilon} \subset \Omega$ is bounded, there exists $c>0$ such that for every $x \in \Omega$ and $\varepsilon_{0}>\varepsilon>0$,

$$
W(x) \geq \frac{c}{1+\left|x-x_{\varepsilon}\right|^{\alpha}},
$$

and on the other hand, for $x \in \Omega \backslash B\left(x_{\varepsilon}, r \varepsilon\right)$, we have

$$
\frac{\varepsilon}{\left|x-x_{\varepsilon}\right|} \leq \frac{2 \varepsilon_{0}}{r \varepsilon_{0}+\left|x-x_{\varepsilon}\right|}
$$

If $r_{0}$ is taken large enough and $\varepsilon_{0}$ is taken small enough, then one has $-\varepsilon^{2} \Delta E_{\varepsilon}(x)+W(x) E_{\varepsilon}(x) \geq 0$.

Remark 42. Observe that when $\alpha=0$, we can also obtain barriers of the form

$$
\exp (-\lambda(|x / \varepsilon|-r))
$$

for some small $\lambda>0$ which provide the decay estimates for the positive solutions of $-\Delta u+u=f(u)$. The restriction $\alpha>0$ in Proposition 41 allows to play with every $\lambda>0$.

The limit case $\alpha=2$ in the exponential barriers yields similarly polynomial barriers of any order.

Proposition 43. Let $N \geq 2, \Omega \subset \mathbb{R}^{N}$ be an unbounded domain and $\left(x_{\varepsilon}\right)_{\varepsilon} \subset \Omega$ be relatively compact in $\Omega$. Assume that $W \in C(\Omega ; \mathbb{R})$ is a positive potential satisfying (7.8) with $\alpha=2$ and

$$
\liminf _{d(x, \partial \Omega) \rightarrow 0} W(x)>0
$$

if $\partial \Omega \neq \emptyset$. Then, for any $\lambda \geq N-2$, there exists $r_{0}>0$ such that the family $\left(P_{\lambda, \varepsilon}\right)_{\varepsilon} \subset H_{\mathrm{loc}}^{1}\left(\Omega \backslash B\left(x_{\varepsilon}, r \varepsilon\right)\right)$ defined by

$$
P_{\lambda, \varepsilon}(x)=\frac{\left(\varepsilon \sqrt{1+r^{2}}\right)^{\lambda}}{\left(\varepsilon^{2}+\left|x-x_{\varepsilon}\right|^{2}\right)^{\lambda / 2}}
$$

is a family of barrier functions for $W$. 


\subsection{Proof of Theorem 30}

To fix the ideas, assume $\Omega$ is a regular exterior domain with nonempty boundary, $\left(\mathcal{G}_{f, \infty}^{1}\right)$ holds and

$$
\liminf _{d(x, \partial \Omega) \rightarrow 0} V(x)>0 .
$$

Let $x_{\varepsilon} \in \Omega$ be such that

$$
u_{\varepsilon}\left(x_{\varepsilon}\right)=\sup _{x \in \Omega} u_{\varepsilon}(x) .
$$

We first claim there exists $\varepsilon_{0}>0$ and $\delta>0$ such that

$$
\inf _{0<\varepsilon<\varepsilon_{0}} d\left(x_{\varepsilon}, \partial \Lambda\right)>\delta \text {. }
$$

Indeed, assume this is not true. Then, we can find sequences $\left(\varepsilon_{n}\right)_{n} \subset \mathbb{R}^{+}$ and $\left(x_{\varepsilon_{n}}\right)_{n} \subset\left(x_{\varepsilon}\right)_{\varepsilon}$ such that $d\left(x_{\varepsilon_{n}}, \partial \Lambda\right) \rightarrow 0$. Going to subsequences if necessary, we now infer that $x_{\varepsilon_{n}}$ converges to some $\bar{x} \in \partial \Lambda$, but this contradicts Proposition 33 and assumption (6.1). We may of course assume that $\delta \leq 1$.

The first statements of the theorem then follows from Proposition 32, Proposition 33 and Proposition 34. Let us now focus on the asymptotic estimate (7.3). Taking $\varepsilon_{0}$ smaller if necessary, we may assume Proposition 36 holds for $\varepsilon_{0}$ and some $r>0$. Now, let $\lambda>0$ and consider the family $\left(E_{\alpha, \lambda, \varepsilon}\right)_{\varepsilon} \subset H_{\text {loc }}^{1}\left(\Omega \backslash B\left(x_{\varepsilon}, r \varepsilon\right)\right)$ of barrier functions associated to the set $\left(x_{\varepsilon}\right)_{\varepsilon}$, provided by Proposition 41. Noticing that the maximum of $u_{\varepsilon}$ is bounded independently of $\varepsilon \leq \varepsilon_{0}$, we deduce from Proposition 39 that

$$
u_{\varepsilon}(x) \leq C \exp \left(\lambda r^{1-\alpha / 2}-\lambda\left|\frac{x-x_{\varepsilon}}{\varepsilon}\right|^{1-\alpha / 2}\right)
$$

in $\Omega$. In particular, since $\left|x-x_{\varepsilon}\right| \geq \delta>0$ for any $x \in \partial \Lambda$, we infer that

$$
u_{\varepsilon}(x) \leq C \exp \left(-\lambda(\delta / \varepsilon)^{1-\alpha / 2}\right)
$$

on $\partial \Lambda$. Therefore, arguing as in the proof of Theorem 22, we now deduce that

$$
u_{\varepsilon}(x) \leq 2 C \exp \left(-\lambda(\delta / \varepsilon)^{1-\alpha / 2}\right) \frac{d(x, \partial \Omega)}{1+d(x, \partial \Omega)},
$$

in $\Omega \backslash \bar{\Lambda}$. Using the facts that the boundary is bounded and $\Lambda \subset \subset \Omega$, and taking (7.9) and (7.10) into account, we finally conclude that

$$
u_{\varepsilon}(x) \leq C \frac{d(x, \partial \Omega)}{1+d(x, \partial \Omega)} \exp \left(-c \lambda\left|\frac{x-x_{\varepsilon}}{\varepsilon}\right|^{1-\alpha / 2}\right)
$$

with $c=\min (1,2 \delta /(\operatorname{diam}(\partial \Omega)+1))^{1-\alpha / 2}$. The estimates $(7.2)$ and $(7.1)$ can be handled in a similar way. 
We emphasize that when considering the growth condition $\left(\mathcal{G}_{f, \infty}^{3}\right)$, we do not have to assume that $V$ is strictly positive up to the boundary. Indeed, the strict positivity of $V$ only plays a role in the construction of the family of exponential and polynomial barrier functions. When $\left(\mathcal{G}_{f, \infty}^{3}\right)$ holds, we have at hand a family of harmonic barriers which can be constructed for any nonnegative potential $V$.

\section{Final comments}

In $[15,17]$, del Pino and Felmer used a penalization scheme to treat the existence of bound state solutions around other type of critical points of the concentration function $\mathcal{C}$. A penalization method is also developed in [16] to catch multi-peak solutions. It could be interesting to find out whether our method can be adapted to those situations.

Another interesting open question concerns the qualitative behaviour of ground states of

$$
-\varepsilon^{2} \Delta u+V(x) u=K(x)|u|^{p-1} u, \quad x \in \mathbb{R}^{N} .
$$

As mentioned earlier, Ambrosetti, Felli and Malchiodi [2] proved the existence of a ground state solution under the assumptions (1.7) and (1.8). If in addition,

$$
\frac{a}{1+|x|^{\alpha}} \leq V(x)
$$

with $0 \leq \alpha<2$, the authors show the ground state belongs to $H^{1}\left(\mathbb{R}^{N}\right)$ and concentrates around a global minimum point of $\mathcal{A}$ as $\varepsilon \rightarrow 0$. Concerning the existence of the ground state solution in a weighted Sobolev space, these conditions can be relaxed by just assuming that $\mathcal{A}$ is coercitive. We then observe that in this case, our result provide the existence of a $H^{1}$ bound state solution for $\varepsilon$ small under less restrictive assumptions on $V$. Namely, if $\left(\mathcal{G}_{\infty}^{3}\right)$ holds and $N>4$, the bound state belongs to $H^{1}\left(\mathbb{R}^{N}\right)$ and concentrates around a global minimum point of $\mathcal{A}$ as $\varepsilon \rightarrow 0$. Such a result is not known for the ground state solution.

\section{References}

[1] Ambrosetti, A., Badiale, M. and Cingolani, S.: Semiclassical states of nonlinear Schrödinger equations. Arch. Rational Mech. Anal. 140 (1997), $285-300$.

[2] Ambrosetti, A., Felli, V. And Malchiodi, A.: Ground states of nonlinear Schrödinger equations with potentials vanishing at infinity. J. Eur. Math. Soc. (JEMS) 7 (2005), 117-144. 
[3] Ambrosetti, A. And Malchiodi, A.: Concentration phenomena for nonlinear Schrödinger equation: recent results and new perspectives. In Perspectives in Nonlinear PDEs, in honor of Haïm Brezis, 19-30. Contemp. Math. 446. Amer. Math. Soc., Providence, RI, 2007.

[4] Ambrosetti, A., Malchiodi, A. and Ni, W.-M.: Singularly perturbed elliptic equations with symmetry: existence of solutions concentrating on spheres. I. Comm. Math. Phys. 235 (2003), 427-466.

[5] Ambrosetti, A., Malchiodi, A. And Ni, W.-M.: Singularly perturbed elliptic equations with symmetry: existence of solutions concentrating on spheres. II. Indiana Univ. Math. J. 53 (2004), no. 2, 297-329.

[6] Ambrosetti, A., Malchiodi, A. and Ruiz, D.: Bound states of nonlinear Schrödinger equations with potentials vanishing at infinity. J. Anal. Math. 98 (2006), 317-348.

[7] Ambrosetti, A. Malchiodi, A. And Secchi, S.: Multiplicity results for some nonlinear Schrödinger equations with potentials. Arch. Ration. Mech. Anal. 159 (2001), 253-271.

[8] Ambrosetti, A. And Ruiz, D.: Radial solutions concentrating on spheres of nonlinear Schrödinger equations with vanishing potentials. Proc. Roy. Soc. Edinburgh Sect. A 136 (2006), no. 5, 889-907.

[9] BAhri, A., Lions, P. L.: On the existence of a positive solution of semilinear elliptic equations in unbounded domains. Ann. Inst. H. Poincaré Anal. Non Linéaire 14 (1997), 365-413.

[10] Bonheure, B. And Van Schaftingen, J. : Nonlinear Schrödinger equations with potentials vanishing at infinity. C. R. Math. Acad. Sci. Paris 342 (2006), no. 12, 903-908.

[11] Byeon, J. AND WANG, Z.-Q.: Standing waves with a critical frequency for nonlinear Schrödinger equations. Arch. Ration. Mech. Anal. 165 (2002), no. 4, 295-316.

[12] Byeon, J. And Wang, Z.-Q.: Standing waves with a critical frequency for nonlinear Schrödinger equations. II. Calc. Var. Partial Differential Equations 18 (2003), no. 2, 207-219.

[13] Byeon, J. And Wang, Z.-Q.: Spherical semiclassical states of a critical frequency for Schrödinger equations with decaying potentials. J. Eur. Math. Soc. (JEMS) 8 (2006), no. 2, 217-228.

[14] Del Pino, M. And Felmer, P.: Local mountain passes for semilinear elliptic problems in unbounded domains. Calc. Var. Partial Differential Equations 4 (1996), 121-137.

[15] Del Pino, M. and Felmer, P.: Semi classical states for Nonlinear Schrödinger equations. J. Funct. Anal. 149 (1997), no. 1, 245-265.

[16] Del Pino, M. And Felmer, P.: Multi-peak bound states for nonlinear Schrödinger equations. Ann. Inst. H. Poincaré Anal. Non Linéaire 15 (1998), no 2, 127-149. 
[17] Del Pino, M. And Felmer, P.: Semi-classical states of nonlinear Schrödinger equations: a variational reduction method. Math. Ann. 324 (2002), no. 1, 1-32.

[18] Floer, A. And Weinstein, A.: Nonspreading wave packets for the cubic Schrödinger equation with a bounded potential. J. Funct. Anal. 69 (1986), 397-408.

[19] Fraenkel, L. E.: An introduction to maximum principles and symmetry in elliptic problems. Cambridge Tracts in Mathematics 128. Cambridge University Press, Cambridge, 2000

[20] Gidas, B., Ni, W. M. And Nirenberg, L.: Symmetry of positive solutions of nonlinear elliptic equations in $\mathbb{R}^{N}$. In Mathematical analysis and applications, Part A, 369-402. Advances in Math. Suppl. Studies 7a. Academic Press, New York-London, 1981.

[21] Grisvard, P.: Elliptic problems in nonsmooth domains. Monographs and Studies in Mathematics 24. Pitman (Advanced Publishing Program), Boston, MA, 1985.

[22] Kufner, A.: Weighted Sobolev spaces. Translated from the Czech. A Wiley-Interscience Publication. John Wiley \& Sons, New York, 1985.

[23] Lions, P. L.: The concentration-compactness principle in the calculus of variations. The locally compact case. II. Ann. Inst. H. Poincaré Anal. Non Linéaire 1 (1984), 223-283.

[24] LI, Y.: Remarks on a semilinear elliptic equation on $\mathbb{R}^{N}$. J. Differential Equations 74 (1988), 34-49.

[25] LI, Y. Y.: On a singularly perturbed elliptic equation. Adv. Differential Equations 2 (1997), 955-980.

[26] Он, Y.-G.: Existence of semiclassical bound states of nonlinear Schrödinger equations with potentials of the class $(V)_{a}$. Comm. Partial Differential Equations 13 (1988), 1499-1519.

[27] OH, Y.-G.: Stability of semiclassical bound states of nonlinear Schrödinger equations with potentials. Comm. Math. Phys. 121 (1989), 11-33.

[28] OH, Y.-G.: On positive multi-bump bound states of nonlinear Schödinger equations under multiple well potential. Comm. Math. Phys. 131 (1990), 223-253.

[29] Opic, B. And Kufner, A.: Hardy-type Inequalities. Pitman Research Notes in Mathematics Series 219. Longman Scientific \& Technical, Harlow, 1990.

[30] Pistoia, A.: Multi-peak solutions for a class of nonlinear Schrödinger equations. NoDEA Nonlinear Differential Equations Appl. 9 (2002), no. 1, 69-91.

[31] Pomponio, A. And Secchi, S.: On a class of singularly perturbed elliptic equations in divergence form: existence and multiplicity results. J. Differential Equations 207 (2004), 229-266. 
[32] Rabinowitz, P.: Minimax methods in critical point theory with applications to differential equations. CBMS Regional Conference Series in Mathematics 65. American Mathematical Society, Providence, RI, 1986.

[33] Rabinowitz, P.: On a class of nonlinear Schrödinger equations. Z. Angew Math Phys 43 (1992), 270-291.

[34] Souplet, P. And Zhang, Q. S.: Stability for semilinear parabolic equations with decaying potentials in $\mathbb{R}^{n}$ and dynamical approach to the existence of ground states. Ann. Inst. H. Poincaré Anal. Non Linéaire 19 (2002), 683-703.

[35] Su, J., Wang, Z.-Q. And Willem, M.: Nonlinear Schrödinger equations with unbounded and decaying potentials. Commun. Contemp. Math. 9 (2007), no. 4, 571-583.

[36] Van Schaftingen, J. and Willem, M.: Symmetry of solutions of semilinear elliptic problems. J. Eur. Math. Soc. (JEMS) 10 (2008), no. 2, 439-456.

[37] WANG, X.: On concentration of positive bound states of nonlinear Schrödinger equations. Comm. Math. Phys. 153 (1993), 229-244.

[38] Wang, X. And Zeng, B.: On concentration of positive bound states of nonlinear Schrödinger equations with competing potential functions. Siam J. Math. Anal. 28 (1997), no. 3, 633-655.

[39] Willem, M.: Minimax theorems. Progress in Nonlinear Differential Equations and their Applications 24. Birkhäuser Boston, Boston, MA, 1996.

Recibido: 4 de octubre de 2006

Denis Bonheure

Université Catholique de Louvain Institut de Mathématique Pure et Appliquée

Chemin du Cyclotron, 2

B-1348 Louvain-la-Neuve, Belgium

denis.bonheure@uclouvain. be

Jean Van Schaftingen

Université Catholique de Louvain

Institut de Mathématique Pure et Appliquée

Chemin du Cyclotron, 2

B-1348 Louvain-la-Neuve, Belgium

jean.vanschaftingen@uclouvain. be

Both authors are supported by the Fonds National de la Recherche Scientifique (Belgium). Jean Van Schaftingen is also supported by the Fonds Spéciaux de Recherche (Université catholique de Louvain). 